\title{
Article \\ Sorafenib Nanomicelles Effectively Shrink Tumors by Vaginal Administration for Preoperative Chemotherapy of Cervical Cancer
}

\author{
Jun Wang ${ }^{1} \mathbb{D}$, Fengmei Lv ${ }^{2}$, Tao Sun ${ }^{2}$, Shoujin Zhao ${ }^{2}$, Haini Chen ${ }^{2}$, Yu Liu ${ }^{1, *}$ and Zhepeng Liu ${ }^{2, *(D)}$ \\ 1 Department of Pharmaceutics, School of Pharmacy, Fudan University \& Key Laboratory of Smart Drug \\ Delivery (Fudan University), Ministry of Education, Shanghai 201203, China; wangjun245186@126.com \\ 2 School of Medical Instrument and Food Engineering, University of Shanghai for Science and Technology, \\ Shanghai 200093, China; LFMusst@126.com (F.L.); suntao930812@163.com (T.S.); shoujinzhao@163.com (S.Z.); \\ HN_C383@163.com (H.C.) \\ * Correspondence: liuyu@fudan.edu.cn (Y.L.); zpliu@usst.edu.cn (Z.L.)
}

Citation: Wang, J.; Lv, F.; Sun, T.; Zhao, S.; Chen, H.; Liu, Y.; Liu, Z. Sorafenib Nanomicelles Effectively Shrink Tumors by Vaginal Administration for Preoperative Chemotherapy of Cervical Cancer. Nanomaterials 2021, 11, 3271. https:// doi.org/10.3390/nano11123271

Academic Editor: Kumar Sudesh

Received: 25 October 2021

Accepted: 29 November 2021

Published: 1 December 2021

Publisher's Note: MDPI stays neutral with regard to jurisdictional claims in published maps and institutional affiliations.

Copyright: (c) 2021 by the authors. Licensee MDPI, Basel, Switzerland. This article is an open access article distributed under the terms and conditions of the Creative Commons Attribution (CC BY) license (https:// creativecommons.org/licenses/by/ $4.0 /)$.

\begin{abstract}
To investigate the potential of sorafenib (SF) in preoperative chemotherapy for cervical cancer to reduce tumor volume, sorafenib micelles (SF micelles) with good stability and high drug loading were designed. SF micelles were prepared by film hydration followed by the ultrasonic method. The results showed that the SF micelles were spherical with an average particle size of $67.18 \pm 0.66 \mathrm{~nm}$ (PDI $0.17 \pm 0.01$ ), a considerable drug loading of $15.9 \pm 0.46 \%(w / w \%)$ and satisfactory stability in buffers containing plasma or not for at least 2 days. In vitro release showed that SF was gradually released from SF micelles and almost completely released on the third day. The results of in vitro cellular intake, cytotoxicity and proliferation of cervical cancer cell TC-1 showed that SF micelles were superior to sorafenib (Free SF). For intravaginal administration, SF micelles were dispersed in HPMC (SF micelles/HPMC), showed good viscosity sustained-release profiles in vitro and exhibited extended residence in intravaginal in vivo. Compared with SF micelles dispersed in N.S. (SF micelles/N.S.), SF micelles/HPMC significantly reduced tumor size with a tumor weight inhibition rate of $73 \%$. The results suggested that SF micelles had good potential for preoperative tumor shrinkage and improving the quality life of patients.
\end{abstract}

Keywords: sorafenib; micelles; cervical cancer; vaginal administration; preoperative chemotherapy

\section{Introduction}

Cervical cancer is a global health problem [1]. Even in developed countries, due to population migration, incomplete screening and limitations of vaccination programs, cervical cancer is still a common gynecological malignant tumor with a high degree of malignancy. Surgery is the main treatment for early-stage cervical cancer, but for patients with intermediate and advanced cervical cancer, the opportunity for surgery is often lost. Therefore, it has now been developed to apply chemotherapeutic drugs before surgery to reduce the size of tumor and improve tumor resection rate [2], to ensure less damage to pelvic tissues, to maintain maximum vaginal length and elasticity and to improve the quality of patient's life under radical hysterectomy [3-6].

At present, cytotoxic treatment of cervical cancer is mainly based on cisplatin combined with chemotherapy. While killing tumor cells, it also kills a large number of normal cells, resulting in a series of toxic side effects [7]. Drugs with targeting properties that improve efficacy while reducing toxic side effects have been a hot spot in recent years for research into the treatment of cervical cancer at home and abroad. VEGF (vascular endothelial growth factor) can directly promote tumor vascular endothelial cell differentiation and proliferation, which is closely related to tumor growth, infiltration and metastasis. Research shows that cervical cancer tissues highly express VEGF [8]. Sorafenib (SF) is a small molecular and multi-target drug. It can inhibit the proliferation of tumor cells by 
blocking RAF/MEK/ERK-mediated cell signal pathway, and also inhibit tyrosine kinase receptors such as VEGF-2 and VEGFR-3 [9]. So, in the current phase I/II clinical trials, tyrosine kinase inhibitors as sorafenib, have been used to inhibit tumor growth and angiogenesis by inhibiting the VEGF signaling pathway $[10,11]$. However, $\mathrm{SF}$ is insoluble in water and has difficulties in delivery. It is clinically prepared as an oral formulation, which limits its application for low bioavailability [12,13] and high dosage [14]. Therefore, it is necessary to develop a new formulation of SF to achieve the ultimate goal of improving water solubility and distribution within tumors.

Researchers have explored many nano-drug deliveries of SF, such as micelles [15-17], liposomes [18-20], polymer nanoparticles [21] and inorganic nanoparticles [22]. However, most of these nanomaterials are not approved by the FDA. D-alpha-Tocopheryl polyethylene glycol 1000 succinate (TPGS) is an FDA certified excipient [23-25]. It is a safe, excellent and amphiphilic nonionic surfactant, which can increase the solubility and the stability of micelles [26]. It can also improve the membrane permeability by inhibiting the efflux of p-gp, increase accumulation and then improve anti-tumor efficacy [27].

Therefore, in this study, SF micelles were prepared using TPGS, which significantly improved the water solubility of SF. HPMC (hydroxypropyl methylcellulose) is a bioadhesive polymer that spreads easily and adheres to the intravaginal to prevent rapid drug loss, by dispersing the SF micelles in the bioadhesive HPMC, prolonged the residence of SF micelles in vagina [28], thereby increasing the distribution of SF in the tumor and improving the therapeutic effect of cervical cancer (Scheme 1).

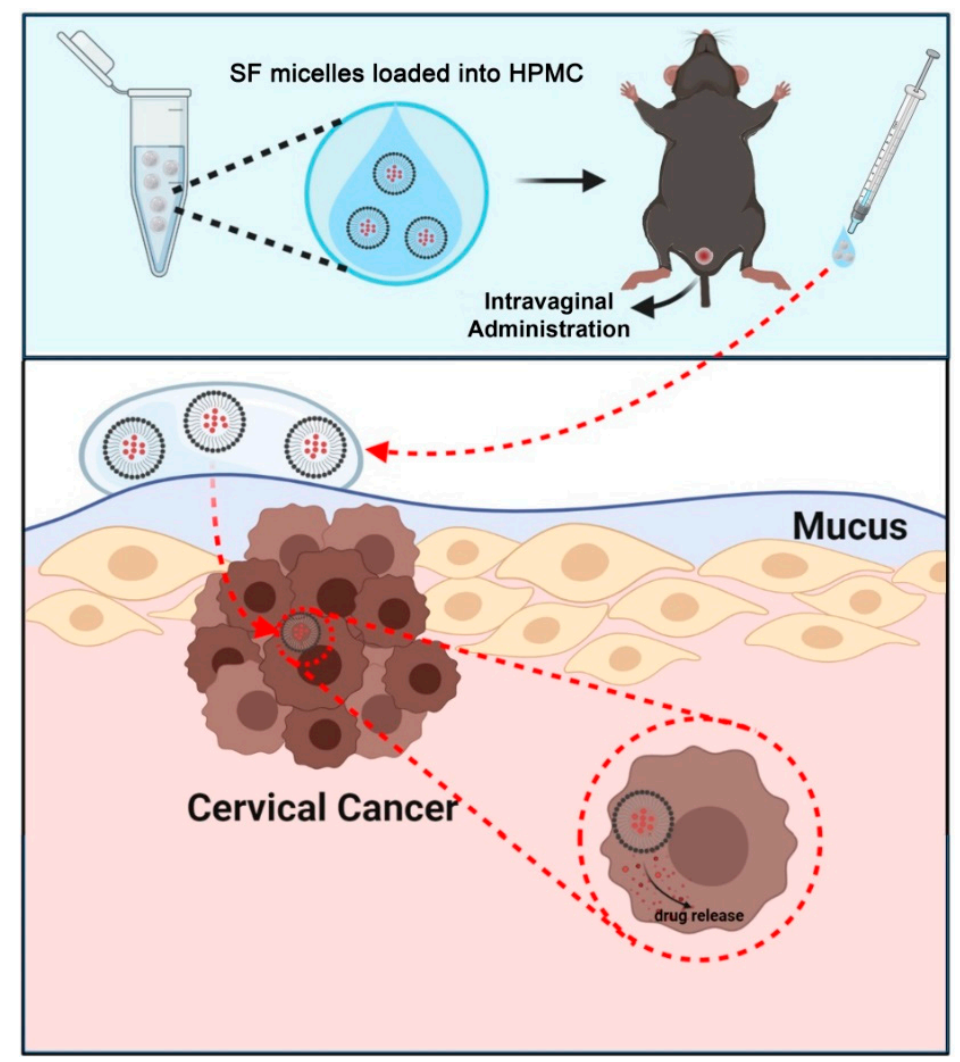

Scheme 1. Treatment of sorafinib micelles dispersed in HPMC after intravaginal administration in an orthotopic mouse cervicovaginal cancer model. Created with BioRender (https://biorender.com/).

\section{Materials and Methods}

\subsection{Materials}

SF, TPGS, hydroxypropyl methylcellulose (HPMC, type I, viscosity of $2 \%(w / w)$ aqueous solution at $20^{\circ} \mathrm{C}: 4000 \mathrm{mPa}$ s), 2-(4-amidinophenyl)-6-indolecarbamindine dihydrochloride (DAPI), 6-coumarin (C6), LysoTracker Red DND-99, DiR iodide, Thiazolyl 
Blue Tetrazolium Bromide (MTT) were purchased from Meilun Biotechnology Co., Ltd. (Dalian, China). All other chemicals were of analytical grade, purchased from Sinopharm Reagent Co., Ltd. (Shanghai, China) and used as received.

TC-1, a cervical cancer-related cell line was purchased from Heyuan Biotechnology Co. Ltd. (Shanghai, China), was cultured in RPMI-1640 complete medium containing 10\% FBS (Gibco, ThermoFisher Co., Waltham, MA, USA), supplemented with $100 \mathrm{U} / \mathrm{mL}$ penicillin and $100 \mu \mathrm{g} / \mathrm{mL}$ streptomycin to prevent cell infection. The humidity of the culture environment was $95 \%$, the temperature was $37^{\circ} \mathrm{C}$, and the $\mathrm{CO}_{2}$ concentration was $5 \%$. Cellular culture dishes, confocal dishes and cell culture plates were purchased from Cellvis (Cellvis, Mountain View, CA, USA).

Female C57BL/ 6 mice (6-8 weeks) were from the Shanghai Laboratory Animal Center (Shanghai, China, production license number SCXK Shanghai 2017-0005) and kept under SPF conditions. Animal experiments were conducted under the guideline approved by the Ethics Committee of the School of Pharmacy, Fudan University (2021-03-SL-LZP-31).

\subsection{Preparation of Micelles}

Dissolve $40 \mathrm{mg}$ TPGS and different masses of SF $(1,1.5,2,4,8,10,15$ and $20 \mathrm{mg})$ in $2 \mathrm{~mL}$ ethanol, and the mixture was mixed well using a vortex, followed by transfer to a $50 \mathrm{~mL}$ eggplant-shaped flask and evaporated in a rotary evaporator (IKA, Staufen, Germany) at defined temperatures $\left(37^{\circ} \mathrm{C}\right)$ using $100 \mathrm{rpm}$ to form a thin film. The rotary evaporation time was about $10 \mathrm{~min}$, and the angle of inclination of the eggplant bottle was adjusted to maximize the contact area between the liquid and the inside of the flask so that the film formed was thin, transparent and homogeneous. Immediately after film formation, $5 \mathrm{~mL}$ of normal saline (N.S., $0.9 \%, w / v$ ) was added and gently mixed at $25^{\circ} \mathrm{C}$ for hydration (the hydration process shall be rapid in order to avoid moisture absorption of the dry film when exposed to air, which would affect the hydration effect), in the meantime the nitrogen blowing needle was dipped into the liquid and the nitrogen flow rate was adjusted to form tiny bubbles during hydration. Finally, the obtained suspension was sonicated in an ice bath for $5 \mathrm{~min}(100 \mathrm{~W})$ to get SF micelles. The method for preparing fluorescently labeled micelles was similar, but in the first step, an additional $25 \mu \mathrm{g} \mathrm{C6}$ or $50 \mu \mathrm{g}$ DiR was added to the $2 \mathrm{~mL}$ ethanol in the eggplant flask.

\subsection{Preparation of Micelles Loaded HPMC}

To increase the sustainability of the micelles in contact with the mucosa and to release the drug slowly, SF micelles dispersed in HPMC (SF micelles/HPMC) were obtained by dissolving an appropriate amount of HPMC $(5 \%, w / w)$ in SF micelles at $25^{\circ} \mathrm{C}$. The specific operation steps were to first weigh $5 \mathrm{~mL}$ of $\mathrm{SF}$, then calculate the mass of HPMC so that HPMC accounts for $5 \%$ of the total mass, and then add HPMC to SF micelles in small amounts several times, stirring ( $200 \mathrm{rpm}$ ) while adding until a uniform translucent gel was formed. DiR-micelles/HPMC were also prepared similarly.

\subsection{Characterization}

\subsubsection{Particle Size and Distribution}

The micelles were diluted with ultrapure water to a SF concentration of $100 \mu \mathrm{g} / \mathrm{mL}$ and then the particle size and zeta potential of micelles were measured at $37^{\circ} \mathrm{C}$ applying the Zetasizer instrument system (ZS-10-82, Malvern Instruments Co., Ltd., Malvern, UK) through dynamic light scattering (DLS) technology.

\subsubsection{Morphology}

The morphology of SF micelles was observed on Tecnai G2F20S-TWIN (FEI Co., Hillsboro, OA, USA) by transmission electron microscopy (TEM). Briefly, $20 \mu \mathrm{L}$ SF micelles with SF concentration of $60 \mu \mathrm{g} / \mathrm{mL}$ was dropped on the copper grid with carbon film attached on the surface. After $15 \mathrm{~min}$, the excess sample was absorbed by the burrs of the filter paper so that there was only a thin layer of the sample on the copper grid without 
droplets. Then the copper lattice was naturally dried at room temperature and observed by TEM.

\subsubsection{Drug Loading (DL) and Encapsulation Efficiency (EE)}

Measurement of UV detection wavelength of SF micelles: SF and TPGS solutions were prepared with acetonitrile at $100 \mu \mathrm{g} / \mathrm{mL}$, respectively, and SF micelles were diluted with acetonitrile until the SF was $50 \mu \mathrm{g} / \mathrm{mL}$ (so that their absorbance values were between 0.2 and 1), and $800 \mu \mathrm{L}$ was placed in a quartz cuvette and the absorption spectra were scanned at $200 \sim 400 \mathrm{~nm}$ by a UV spectrophotometer to determine the maximum absorption wavelength.

The freeze-dried SF micelles were accurately weighed and dissolved in an appropriate volume of acetonitrile to make the concentration within the linear range. After filtered with a $0.22 \mu \mathrm{m}$ syringe filter, the filtrate was analyzed by an Agilent 1100 HPLC system (Palo Alto, CA, USA). The HPLC condition was: Diamonsil ${ }^{\circledR} \mathrm{C} 18$ column (250 mm $\times 4.6 \mathrm{~mm}, 5 \mu \mathrm{m}$, Dikma Technology, Beijing, China), $\mathrm{MeOH}: \mathrm{KH}_{2} \mathrm{PO}_{4}(\mathrm{pH}=2.5$, 70:30, v:v), $0.7 \mathrm{~mL} / \mathrm{min}, 262 \mathrm{~nm}$. DL and EE were calculated using the following equations.

$$
\begin{gathered}
\mathrm{EE}(\%)=\frac{\text { Amount of drug in micelles }}{\text { Total amount of feeding drug }} \times 100 \\
\mathrm{DL}(\%)=\frac{\text { Amount of drug in micelles }}{\text { Total amount of micelles }} \times 100
\end{gathered}
$$

\subsubsection{Crystalline Characterization}

The SF micelle lyophilized powder was analyzed by X-ray powder diffraction (XRPD) using a D2 Phaser diffractometer (BrukerCorp, Billerica, MA, USA). Test conditions: The voltage was $40 \mathrm{kV}$. The current was $100 \mathrm{~mA}$. The rotation range of the diffraction angle $(2 \theta)$ was 0 to $50^{\circ}$, the scanning speed was $5^{\circ} / \mathrm{min}$, and the step size was $0.02^{\circ}$.

Differential scanning calorimeter (DSC) analysis of micelles and bulk drugs was measured by a Perkin-Elmer Pyris 1 DSC instrument (Waltham, MA, USA) with an Intracooler 2P cooling accessory. Preweighted lyophilized powders were sealed into aluminum crucible (NETZSCH, Selb, Germany) and place an empty aluminum crucible as a reference. The nitrogen flow rate was $10 \mathrm{~mL} / \mathrm{min}$, the heating rate was $10^{\circ} \mathrm{C} / \mathrm{min}$, and the scanned temperature ranged from 20 to $300^{\circ} \mathrm{C}$.

\subsubsection{Stability}

The time-dependent stability of SF micelles in different media including PBS, RPMI 1640 medium containing $10 \%$ FBS, and FBS were evaluated for $48 \mathrm{~h}$ (concentration of SF equivalent to $300 \mu \mathrm{g} / \mathrm{mL}$ ). At each time point, $200 \mu \mathrm{L}$ micelles in different media were taken out and the particle size was measured using the DLS, while another $200 \mu \mathrm{L}$ was removed to monitor aggregation by detect their absorbance at $560 \mathrm{~nm}(560 \mathrm{~nm}$ as the turbidity characteristic wavelength) using the Synergy 2 (Biotek, Green Mountains, VT, USA).

\subsubsection{In Vitro Release}

In vitro release behavior of SF micelles were detected by bag filter method with vaginal fluid simulant (VFS) [29] containing 1\% Tween $80(w / w)$ as the release medium. Briefly, $1 \mathrm{~mL}$ SF micelles or SF bulk (SF concentration $500 \mu \mathrm{g} / \mathrm{mL}$ ) were added into SnakeSkin ${ }^{\mathrm{TM}}$ dialysis bags with MWCO 35000Da (ThermoFisher, Waltham, MA, USA), and then completely immersed into $10 \mathrm{~mL}$ release medium and continuously shook under $100 \mathrm{rpm}$ by a reciprocating shaker bath (THZ-103B, Shanghai Hengyi Scientific Instruments Co. Ltd., Shanghai, China) at $37^{\circ} \mathrm{C} .400 \mu \mathrm{L}$ samples of different groups were taken from the medium at predetermined time intervals and replaced with an equal volume of fresh medium, and the released SF concentration was analyzed by HPLC to obtain the cumulative release percentage. HPLC conditions were similar to Section 2.4.3. 


\subsection{Cellular Experiments}

\subsubsection{Cellular Uptake}

The uptake of SF micelles by cervical cancer cells was carried on TC-1 cells, which exhibited similar genetic traits to cervical tumors induced by human papillomavirus (HPV) [30]. For fluorescent imaging, TC-1 cells were inoculated onto the 12-hole plate at the density of $2 \times 10^{5}$ cells per hole. After $12 \mathrm{~h}$ incubation, the medium was replaced with fresh medium containing C6-labeled SF micelles and Free C6 (the terminal concentration of C6 was $10 \mathrm{nM}$ ). After $4 \mathrm{~h}$ incubation, the medium was removed and the cells were washed twice with PBS to avoid the interference of unbound SF micelles. We treated the cells with $4 \%$ paraformaldehyde fix solution for $15 \mathrm{~min}$, stained the nulei with DAPI for $3 \mathrm{~min}$ and finally took fluorescence images with a fluorescence microscope (Leica, DMI4000 B, Wetzlar, Germany).

For colocalization with lysosomes, cells which had been incubated with C6-labeled SF micelles and Free C6 were treated with $37^{\circ} \mathrm{C}$ preheated LysoTracker Red DND-99 (50 nM) for $45 \mathrm{~min}$ and then fixed with paraformaldehyde fix solution for $15 \mathrm{~min}$, finally treated with DAPI for 3 min to track its position. Fluorescent images were captured by Leica confocal microscope (Leica, TCS SP8, Germany). The excitation (Ex) and emission (Em) parameters were: DAPI Ex $364 \mathrm{~nm} / \mathrm{Em} 461 \mathrm{~nm}, \mathrm{C} 6$ Ex $466 \mathrm{~nm} / \mathrm{Em} 504 \mathrm{~nm}$ and LysoTracker Red Ex $577 \mathrm{~nm} / \mathrm{Em} 590 \mathrm{~nm}$. The colocalization coefficient of fluorescence was analyzed by ImageJ software (Image J 2x 2.1.4.7 Wayne Rashand, National Institutes of Health, Bethesda, MD, USA).

For flow cytometry, the process was similar to the above cell uptake steps. The difference was that the cells were digested with trypsin and then collected by centrifugation (1000 rpm, $10 \mathrm{~min}$ ) after being washed twice with PBS. The collected C6 labeled cells were finally resuspended in PBS and counted by a flow cytometer BD FACSCalibur Flow Cytometry System (Applied Cytometry Systems, Franklin Lakes, NJ, USA).

\subsubsection{In Vitro Cytotoxicity}

The TC- 1 cells were seeded into a 96-well plate at a seeding density of $3 \times 10^{3}$ cells per well. After $12 \mathrm{~h}$ incubation, the cells were treated with fresh medium, to which SF micelles and Free SF were added to provide the final concentration of SF equivalent to $12.91 \mu \mathrm{M}, 17.21 \mu \mathrm{M}, 20.65 \mu \mathrm{M}, 32.27 \mu \mathrm{M}, 43.03 \mu \mathrm{M}, 51.63 \mu \mathrm{M}$ and $64.54 \mu \mathrm{M}$, respectively. After $24 \mathrm{~h}$ incubation, cell viability was measured by MTT method. Briefly, TC-1 cells were treated with MTT ( $5 \mathrm{mg} / \mathrm{mL}, 20 \mu \mathrm{L}$ per well) for $4 \mathrm{~h}$. The medium was removed with a $5 \mathrm{~mL}$ syringe and the formazan crystal was fully dissolved in dimethyl sulfoxide (DMSO). Absorbance data was performed by Power Wave XS Microplate Spectrophotometer at 490 nm (BioTek Instruments, Inc., Winooski, VT, USA).

Preparation of free sorafenib solution: since sorafenib is almost insoluble in water, we followed the formulation of $\mathrm{Taxol}^{\circledR}$ in this experiment [31], that is, a mixture of polyoxyethylene castor oil: ethanol $=1: 1(\mathrm{v}: \mathrm{v})$ to dissolve sorafenib, and after sonication to obtain a free sorafenib solution (Free SF).

\subsection{Characterization of HPMC as a Micellar Dispersion Carrier}

HPMC was added to the SF micelles to a mass fraction of $5 \%$ and then took $1 \mathrm{~mL}$ and placed it directly under the rotor (this process should be done as slowly as possible to avoid bubbles in the sample, and extra sample could be scraped off using a flat scraper) and tested for elastic $\left(G^{\prime}\right)$ and viscous modulus $\left(G^{\prime \prime}\right)$ by rheology (Bohlin Gemini 2, Malvern Panalytical, Malvern, UK). The parameters were as follows: Mode: oscilllation, Temperature: $25^{\circ} \mathrm{C}$, Frequency: $1 \mathrm{~Hz}$, Measuring System: PP40 Acrylic, the correlation curves of $\mathrm{G}^{\prime}$ and $G^{\prime \prime}$ vs. strain were obtained at the end of the measurement.

\subsection{In Vivo Intravaginal Residence}

$10 \mu \mathrm{L}$ DiR-labelled micelles dispersed in HPMC (DiR-micelles/HPMC) or saline (DiRmicelles/N.S.) was intravaginally administered into healthy female ICR mice by operating 
a microliter syringe equipped with a smooth blunt needle as previously reported [32]. Fluorescence images were taken at $0,1,3$ and $5 \mathrm{~h}$ after administration with a fluorescence in vivo imaging system (IVIS Spectrum, PerkinElmer, Santa Clara, CA, USA) at Ex $748 \mathrm{~nm} / \mathrm{Em} 780 \mathrm{~nm}$.

\subsection{In Vivo Evaluation in TC-1-Luc Model Tumor-Bearing Mice}

Six- to eight-week-old female C57BL/ 6 mice were purchased from Shanghai Slack Laboratory Animal Co., Ltd. (license number: production license number SCXK Shanghai 2017-0005) and housed in an SPF animal room. All animal experiment conforms to the ethical standards of animal experiment and was approved by the Experimental Animal Ethics Committee of the School of Pharmacy of Fudan University. The orthotopic cervical model of C57BL/ 6 mice were established referring to the method of Ci et al. [33]. Mice were subcutaneously injection with $\beta$-estradiol ( $0.1 \mathrm{mg} /$ day) for a successive seven days before tumor inoculation. Then mice were anesthetized by intraperitoneal injection of $8 \%$ chloral hydrate $(w / v, 100 \mu \mathrm{L}$ per mouse) and placed them in a supine position. Finally, sterilized dry cotton balls were moistened with VFS to softly wipe off vaginal secretions, and a sterilized cytobrush was used to gently disrupt the cervicovaginal epithelium. TC1-Luc cells were diluted to required concentration and then inoculated intravaginally at $1 \times 10^{5}$ cells per mouse. To avoid a drop in body temperature during anesthesia, the mice were placed on an insulating pad to keep them warm until they awoke and then placed back in their cages. The successful establishment of the tumor model was confirmed by palpation, anatomical and histological examination. When it was found that TC-1 tumors volume grew to about $100 \mathrm{~mm}^{3}$, it was defined as day 0 and intravaginal administration was started. The mice were anesthetized at a low dose after administration and kept in a supine position for $20 \mathrm{~min}$ to avoid the mice licking. We employed intravaginal administration because local administration for cervical cancer could effectively release the drug at the lesion site and allow full play to its anti-tumor effect [34-36]. We used a $10 \mu \mathrm{L}$ syringe equipped with a blunt needle for intravaginal administration, and the needle was dipped into the VFS before administration to obtain lubricity and to reduce mucosal damage as well as the pain to the mice when the needle entered and exited the vagina.

Mice were randomly divided into five treatment groups ( $n=6 /$ group) and intravaginally treated with N.S., HPMC, empty micelles, Free SF/HPMC $(15 \mathrm{mg} / \mathrm{kg})$ and SF micelles/HPMC (15 mg/kg). Since HPMC was insoluble in ethanol, Free SF/HPMC was prepared in in vivo anti-tumor experiments by first dissolving SF with DMSO, then diluting it with ultrapure water to the required concentration for in vivo administration, and finally adding HPMC in small amounts several times to obtain Free SF/HPMC. The drug was administered intravaginally every 3 days, and the body weight and tumor bioluminescence intensity were monitored. D-Luciferin at $150 \mathrm{mg} / \mathrm{kg}$ was injected intraperitoneal into the tumor of TC-1-Luc cervical cancer model $10 \mathrm{~min}$ prior to whole-body imaging. Use the Xenogen IVIS ${ }^{\circledR} 200$ Series to obtain whole-body bioluminescence images, and the region of interest (ROI) was acquisition and quantification applying Living Image ${ }^{\circledR}$ software. On day 16 , mice were sacrificed to resecting the tumors connecting uterus and uterine tubes for photographing (to prove that they were orthotopic tumors), weighing the tumors and further H\&E (Hematoxylin-eosin) microscopic examination of tumors, vaginas and organs. The relative bioluminescence intensity was calculated as Formula (3) and the inhibition rate of tumor weight was calculated as Formula (4):

$$
\begin{gathered}
\text { Relative bioluminescence intensity }=\frac{\mathrm{V}_{\text {bioluminescence intensity on the day of measurement }}}{\mathrm{V}_{\text {Bioluminescence fluorescence intensity on day } 0}} \times 100 \% \\
\text { Inhibition rate of tumor weight }=\frac{\mathrm{W}_{\text {saline group }}-\mathrm{W}_{\text {treatment group }}}{\mathrm{W}_{\text {saline group }}} \times 100 \%
\end{gathered}
$$




\subsection{Statistical Analysis}

All statistical analysis was performed using GraphPad Prism 8 (La Jolla, CA, USA). The statistical differences among groups were conducted by the two-way ANOVA test. A value of $p<0.05$ was considered significant and $p<0.01$ was considered highly significant.

\section{Results and Discussion}

\subsection{Optimization}

SF micelles were prepared by thin-film hydration method [37], and SF micelles with different ratios of SF:TPGS were placed in small glass bottles to observe the Tyndall effect. The beam passed through all different ratios (Figure A1), indicating that the fresh SF micelles were uniformly dispersed in water. The best ratio of SF to TPGS was further optimized by particle size, polydispersity (PDI), zeta-potential, drug loading (DL) and entrapment efficiency (EE). The results were shown in Table A1, with the increase of the SF from 1:40 to 8:40, the drug loading of SF in micelles increased from $1.91 \%$ to $15.9 \%$, and the average particle size increased from less than $40 \mathrm{~nm}$ to $67 \mathrm{~nm}$ (Figure A2), which was due to the mass of TPGS remained constant and as the SF increased, more SF was loaded into the core of the micelles, thus increasing the particle size and drug loading. The encapsulation efficiency was decreased but not significantly different, all being greater than $89 \%$. It was worth mentioning that with the increased of SF from 1:40 to 4:40, the particle size did not gradually increase which might be due to the excessive TPGS forming small empty micelles, which interfere with the particle size results. While continue to increase the SF content to a ratio higher than 8:40, the encapsulation efficiency dropped sharply, probably because the drug has exceeded the carrier TPGS encapsulation. Whereas the drug loading still increased probably possibly due to some of the SF formed nanocrystals with a small amount of TPGS, which was a thermally unstable system with high drug loading [38], hence the drug loading increased. However, due to nanocrystal instability, 10:40, 15:40 and 20:40 all precipitated after being stored at room temperature for $2 \mathrm{~h}$, therefore SF:TPGS = 8:40 (1:5) was selected for the following experiment.

\subsection{Successful Preparation of SF Micelles}

SF micelles with optimized ratio 1:5 (SF:TPGS) were successfully prepared as shown in Figure 1. The SF micelles exhibited a near-spheroid shape with a size of $67.18 \pm 0.66 \mathrm{~nm}$ (Figure 1A). The zeta potential of SF micelles was $-1.54 \pm 0.8 \mathrm{mV}$ (Figure 1B). UV spectrum result displayed that SF and TPGS had the maximum ultraviolet absorption wavelengths at $205 \mathrm{~nm}$ and $262 \mathrm{~nm}$ (Figure A3), respectively, SF micelles had absorption wavelengths for both, since the large difference between $205 \mathrm{~nm}$ and $262 \mathrm{~nm}$, the presence of TPGS would not interfere SF measurements. The DL and EE of SF micelles measured by HPLC at $262 \mathrm{~nm}$ were $15.9 \pm 0.46 \%$ and $89.4 \pm 0.19 \%$, respectively (Table A1).

In the XRPD spectrum (Figure 1D), bulk SF displayed diffraction peaks at $10.7^{\circ}$, $19.1^{\circ}, 22.8^{\circ}, 23.3^{\circ}$ and $25^{\circ}$, bulk TPGS displayed diffraction peaks at $8.5^{\circ}, 9.0^{\circ}, 11.0^{\circ}, 17.0^{\circ}$ and $17.7^{\circ}$, the physical mixture of bulk SF and bulk TPGS showed both two separate characteristic peaks, while SF micelles showed diffraction peaks at $29.10^{\circ}$ and $33.50^{\circ}$, indicating that the crystallinity structure of SF micelles was different from that of bulk SF, and SF micelles were not a simple physical mixture of SF and TPGS. DSC further verified their difference in crystal structures (Figure 1E), bulk SF and bulk TPGS displayed an exothermic peak at about $217^{\circ} \mathrm{C}$ and $107^{\circ} \mathrm{C}$, respectively, and the physical mixture of bulk $\mathrm{SF}$ and bulk TPGS remained the endothermic peak of bulk SF, while there was no such characteristic peak in SF micelles, but displayed an endothermic peak around $60^{\circ} \mathrm{C}$, which suggested that mixing SF and TPGS might not change the crystalline of SF; however, the formulation SF into SF micelles, the crystalline was changed. 


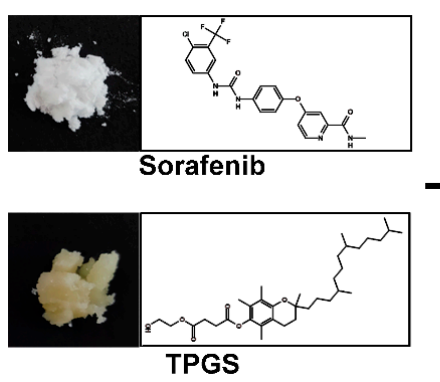

A
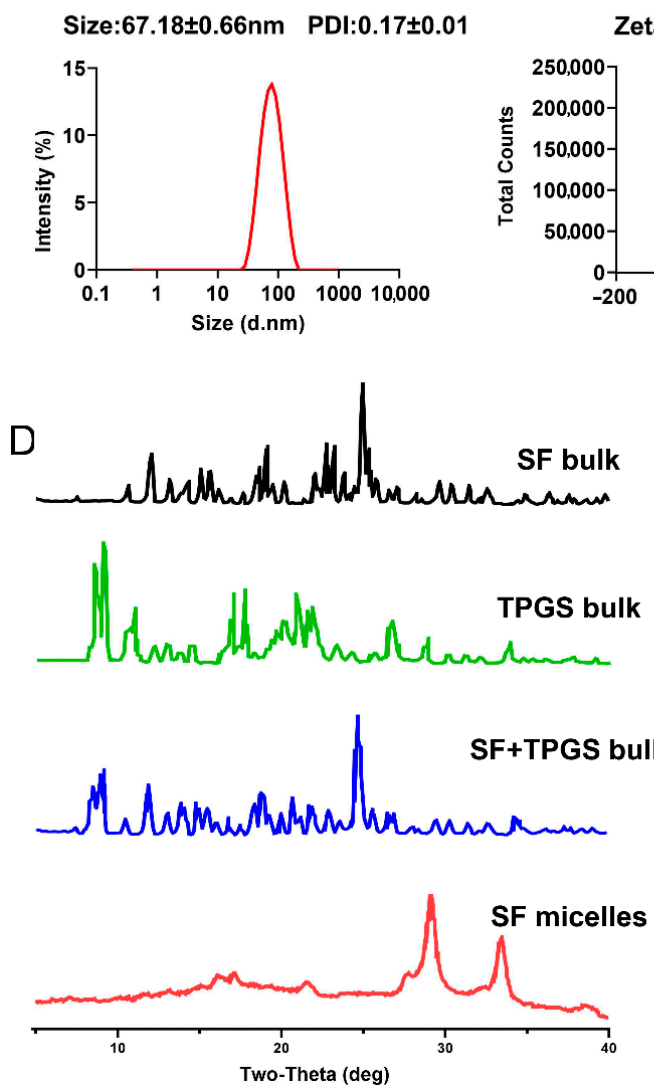

B

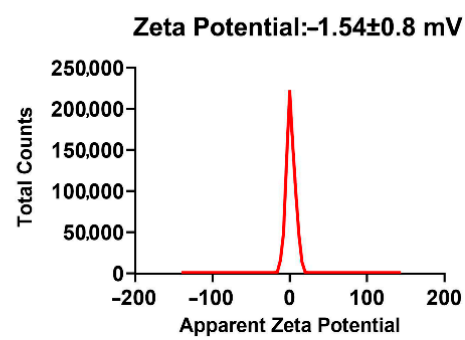

C

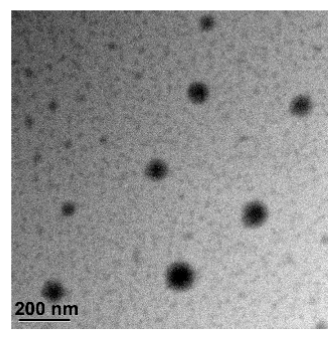

$E$

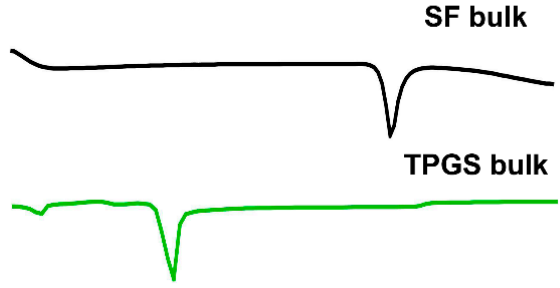

SF+TPGS bulk
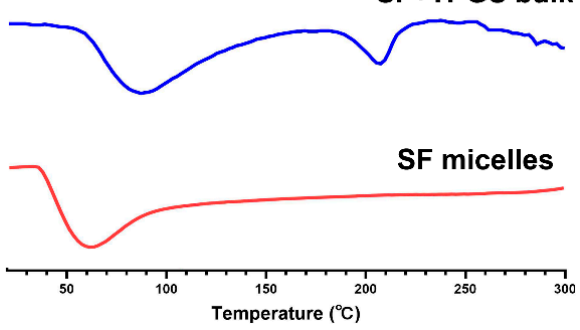

Figure 1. SF micelles characterization. (A) Size distribution and (B) zeta-potential of SF micelles. (C) TEM image (bar = $200 \mathrm{~nm})$. (D) XRPD patterns. (E) DSC curves.

From Figure 2A, the stability of SF micelles was evaluated in different mediums including $0.01 \mathrm{M}$ PBS, RPM1640 + 10\% FBS, and FBS, respectively. The particle size results measured by DLS showed that micelles could be stable for at least $48 \mathrm{~h}$ in all three media, while the particle size measured in RPM1640 + 10\% FBS and FBS media was larger than that measured in PBS, which might be due to the protein crown formed by FBS on the particle surface, thus we further used the previously reported method to assess the agglomeration of particles in the presence of FBS by measuring the absorbance of micelles in different media at $560 \mathrm{~nm}$ [39-42]. The results indicated that the SF micelles remained good stability in both PBS and serum-containing PBS buffer (Figure 2C). 

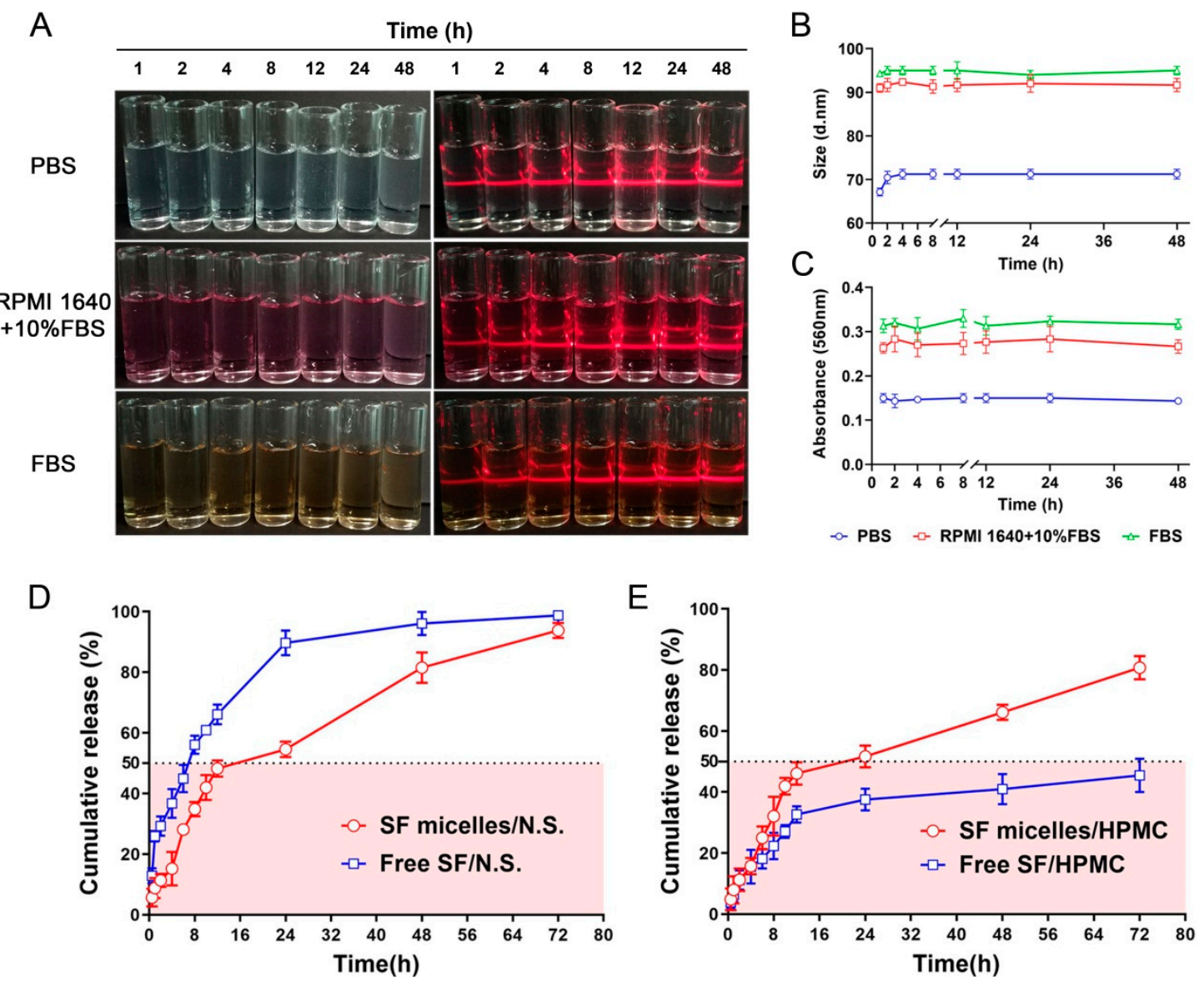

Figure 2. Stability and release profiles of SF micelles. (A) Photographs of SF micelles after incubation in different mediums including PBS, RPMI 1640 medium containing 10\% FBS and FBS. (B) Size change in different mediums measured by DLS. (C) Size aggregation in different mediums assessed by absorbance at $560 \mathrm{~nm}$. (D) In vitro release of SF micelles dispersed in N.S. and (E) HPMC in VFS containing $1 \%$ Tween $80(w / v)$ at $37^{\circ} \mathrm{C}(\mathrm{n}=3$, mean \pm SD).

Compared with Free SF, SF micelles had a slow-release effect and could be completely released within $72 \mathrm{~h}$ (Figure 2D), providing evidence for the dosing interval in subsequent animal experiments (every 3 days). Compared with the release properties of SF micelles in saline (SF micelles/N.S.), the sustained release effect of SF micelles loaded with HPMC (SF micelles/HPMC) was more obvious. It took about $12 \mathrm{~h}$ for SF micelles/N.S. while about $24 \mathrm{~h}$ for SF micelles/HPMC to reach a cumulative release of $50 \%$. We observed that the cumulative release of Free SF/HPMC reached a plateau at about $24 \mathrm{~h}$ and was less than $50 \%$ at $72 \mathrm{~h}$, which might be due to the precipitation of Free SF in HPMC.

\subsection{In Vitro Cellular Uptake and Cytotoxicity of Micelles}

For fluorescent images, SF didn't have fluorescent properties and could not track its uptake behavior in cells. Therefore, a general fat-soluble fluorescent C6 (Free C6) was selected to represent free sorafenib $[43,44]$. As shown in Figure 3A-C, cellular uptake of Free $\mathrm{C} 6$ was less than that of SF micelles. The intracellular fluorescence in cells was stronger after incubated with C6-labeled SF micelles and most of which located around the nucleus and overlapped with lysosomal fluorescence, while the fluorescence of Free C6 was much weaker and seemed scattered in the cytoplasm, and only a small part of it overlapped with the lysotracker. (Figure 3D-F). 
A
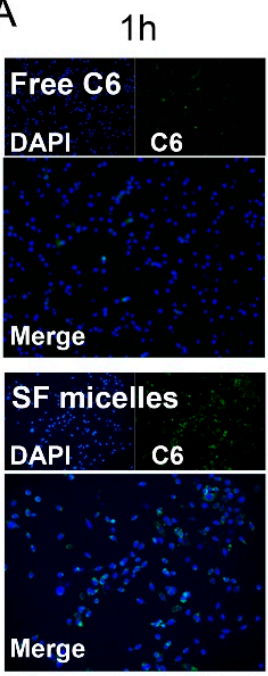

D

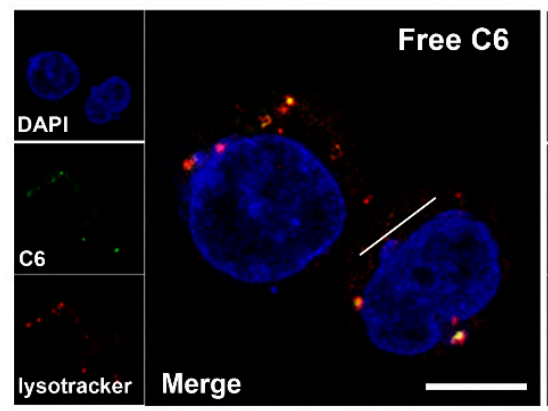

$2 \mathrm{~h}$
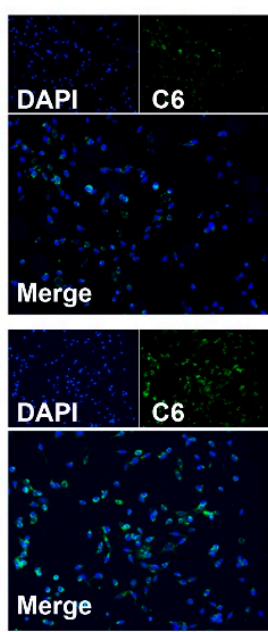

Mèrge
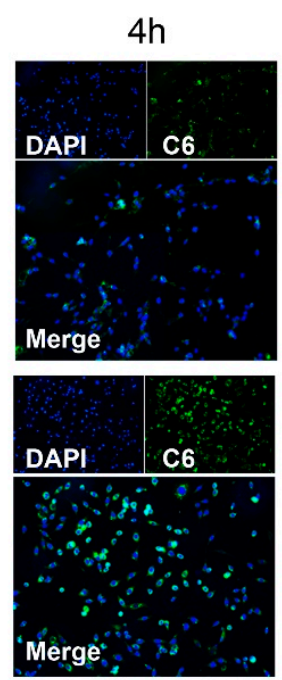

B

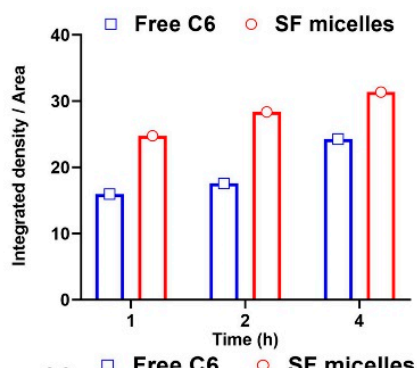

C

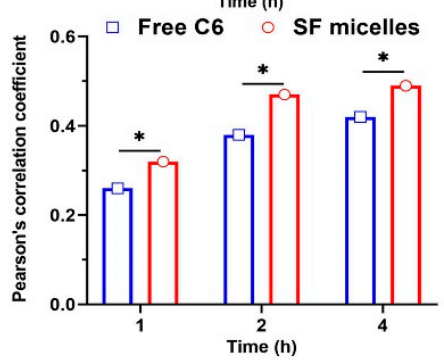

E
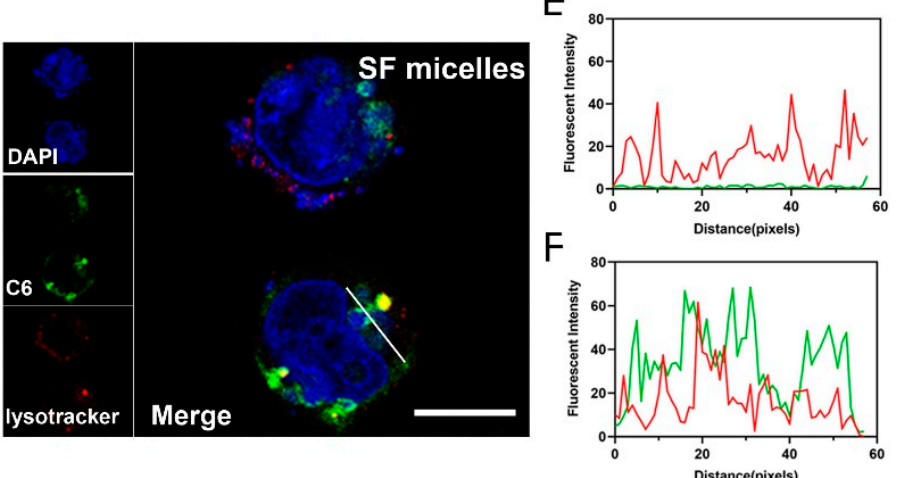

Figure 3. Interaction between SF micelles and TC-1 cells. (A) Fluorescent microscopic photographs of TC-1 cells after $1 \mathrm{~h}, 2 \mathrm{~h}$ or $4 \mathrm{~h}$ incubation with Free C6 and C6-labeled SF micelles (green). The nucleus was stained with DAPI (blue). (B) The Average fluorescence intensity (fluorescence intensity/Area) and (C) corresponding co-localization profiles of photographs in Figure A were calculated by Image J software (The Pearson's correlation coefficient represents the colocalization between the nucleus (blue) and Free C6 and SF micelles (green). Higher values represent a stronger degree of co-location). (D) Representative intracellular localization images of C6-labeled SF micelles incubated with TC-1 cells with $4 \mathrm{~h}$. The lysosomes stained by Lysotracker were red and the nucleus stained by DAPI were blue. The bar is $20 \mu \mathrm{m}$. (E) The corresponding co-localization profiles of Free C6 and (F) SF micelles in Figure D were calculated by Image J software. The red line stood for lysosomes, and the green line stood for micelles. Significance criteria: ${ }^{*} p<0.05$.

Cytotoxicity was evaluated at different concentrations of SF in SF micelles and Free SF. After $24 \mathrm{~h}$ incubation, $\mathrm{IC}_{50}$ of Free SF and SF micelles was $76.65 \mu \mathrm{M}$ and $46.76 \mu \mathrm{M}$, respectively. SF micelles exhibited a significant antiproliferative effect on tumor cells at low concentrations. It was worth mentioning that empty micelles had no obvious cytotoxicity at all concentrations, which proved the safety of TPGS (Figure 4A). Flow cytometry (Figure 4B) revealed higher cellular uptake of SF micelles than that of Free C6 after $4 \mathrm{~h}$ co-incubation as well. The apoptosis results showed that cells treated with empty micelles for $24 \mathrm{~h}$ had no obvious apoptosis, demonstrating the good biocompatibility of TPGS, while both Free SF and SF micelles had apoptotic effects on the cells with apoptosis rates of $10.12 \pm 0.49 \%$ and $23.76 \pm 1.37 \%$, respectively (Figure $4 \mathrm{C}, \mathrm{D}$ ). It indicated that SF micelles could induce apoptosis in TC-1 cells to some extent. 
A

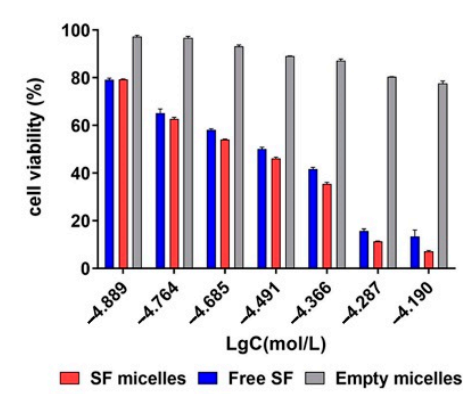

$\mathrm{D}$

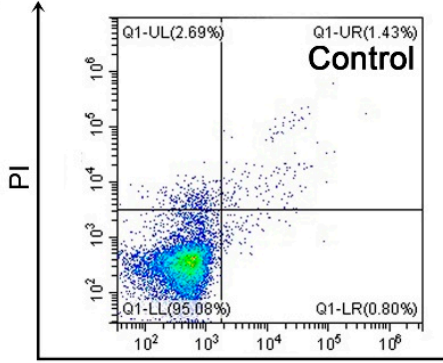

B

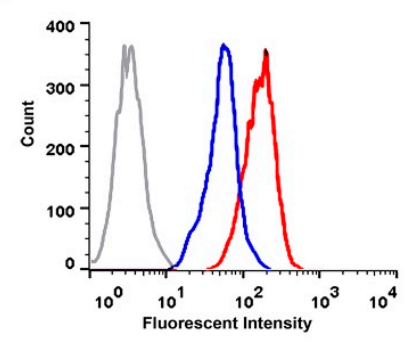

- SF micelles - Free SF - Empty micelles

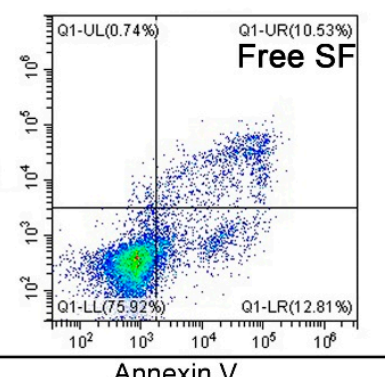

C

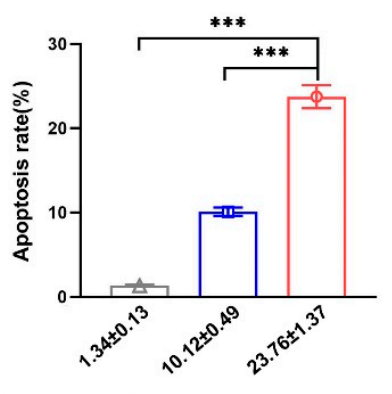

SF micelles $\square$ Free SF $\triangle$ Empty micelles

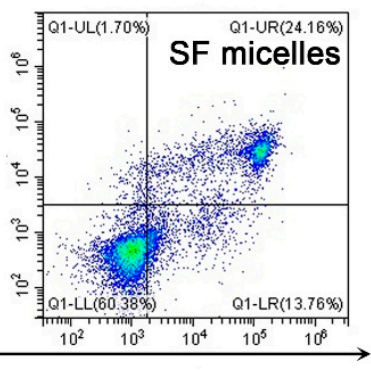

Figure 4. Cellular uptake and cytotoxicity in TC-1 cells. (A) In vitro cytotoxicity of Free SF, Empty micelles, or SF micelles after $24 \mathrm{~h}$ of co-incubation in TC-1 cells $(n=3$, mean \pm SD). (B) Flow cytometry analysis on the fluorescent intensity of cells after co-incubation for $4 \mathrm{~h}$ with Free SF and C6-labeled Empty micelles or SF micelles. (C) Cell apoptosis were measure by flow cytometry with Annexin V-FITC and propidium iodide (PI) staining after co-incubation with Free SF, Empty micelles, or SF micelles in TC-1 cells ( $n=3$, mean \pm SD). (D) Typical apoptotic cell images. Significance criteria: *** $p<0.001$.

\subsection{SF Micelles Dispersed in HPMC Facilitate Intravaginal Retention}

For in vivo experiences, SF micelles were dispersed in HPMC to avoid immediate leakage caused by administered as an aqueous dispersion. The adhesion of HPMC enhanced the contact between micelles and mucosa, so that the SF had time to be absorbed and achieve the purpose of treatment. HPMC was a practical vaginal administration vehicle with the advantages of safety, simple preparation process and good drug compatibility [45]. A 5\% $(w / w)$ concentration of HPMC was selected to deliver SF micelles. The elastic $\left(\mathrm{G}^{\prime}\right)$ and viscous moduli $\left(G^{\prime \prime}\right)$ confirm the adhesion of HPMC. As shown in Figure 5, the $G^{\prime}$ and $\mathrm{G}^{\prime \prime}$ of SF micelles dispersed in N.S. (SF micelles/N.S.) were less than $1 \mathrm{~Pa}$, while the $\mathrm{G}^{\prime}$ and $G^{\prime \prime}$ of SF micelles dispersed in HPMC (SF micelles/HPMC) increased to more than 1000 pa. We also monitored the particle size variation of SF micelles loaded into HPMC. Before measuring the particle size of SF micelles/HPMC with DLS, it was diluted with ultrapure water to obtain good fluidity and solution-like state, and then the particles were fully dispersed by bath ultrasonic for $30 \mathrm{~s}$. The results showed an increase from $67 \mathrm{~nm}$ to about $178 \mathrm{~nm}$ (Figure A4), which was still conducive to penetrating mucus due to its nanoscale less than $500 \mathrm{~nm}[46,47]$.

Before intravaginal administrated DiR-micelles/HPMC and DiR-micelles/N.S., vaginals were wash with VFS to achieve the purpose of cleaning. Fluorescence photos and semi-quantitative results indicated that SF micelles/HPMC has a prolonged residence (Figure 6). 

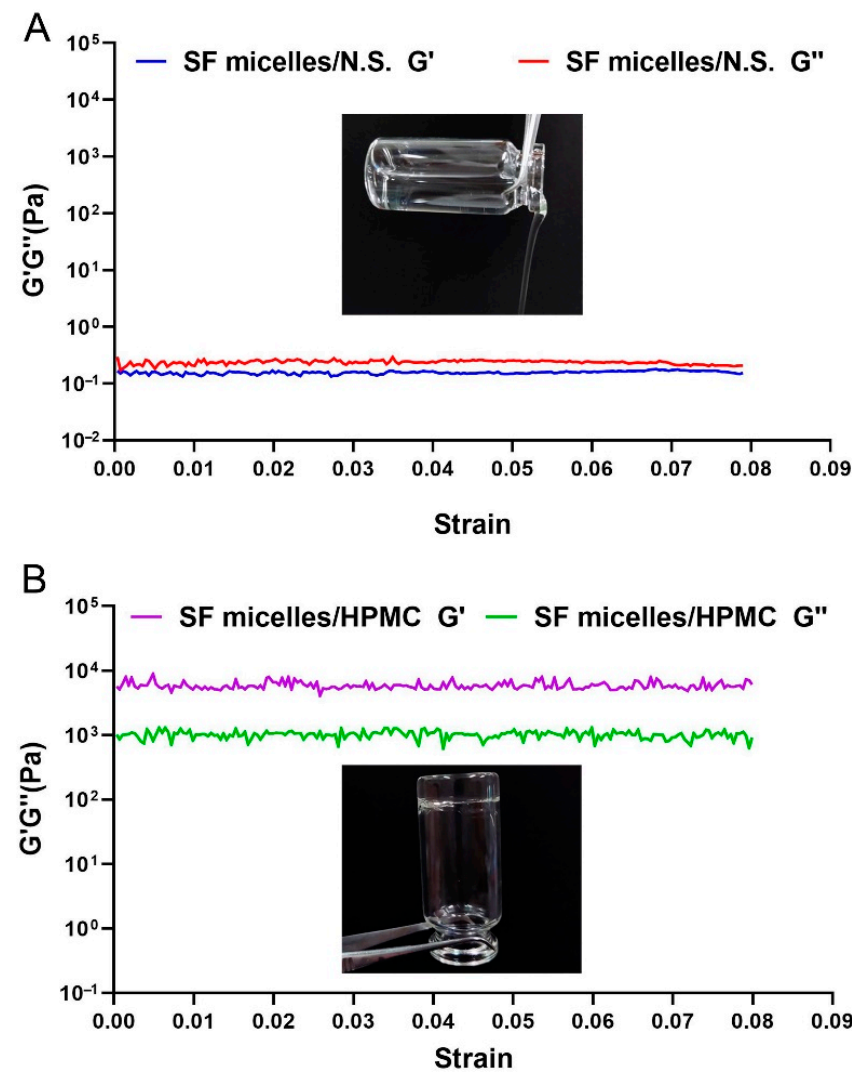

Figure 5. Frequency spectra of the elastic $\left(G^{\prime}\right)$ and viscous moduli $\left(G^{\prime \prime}\right)$ of $(A)$ SF micelles dispersed in N.S. and (B) HPMC.
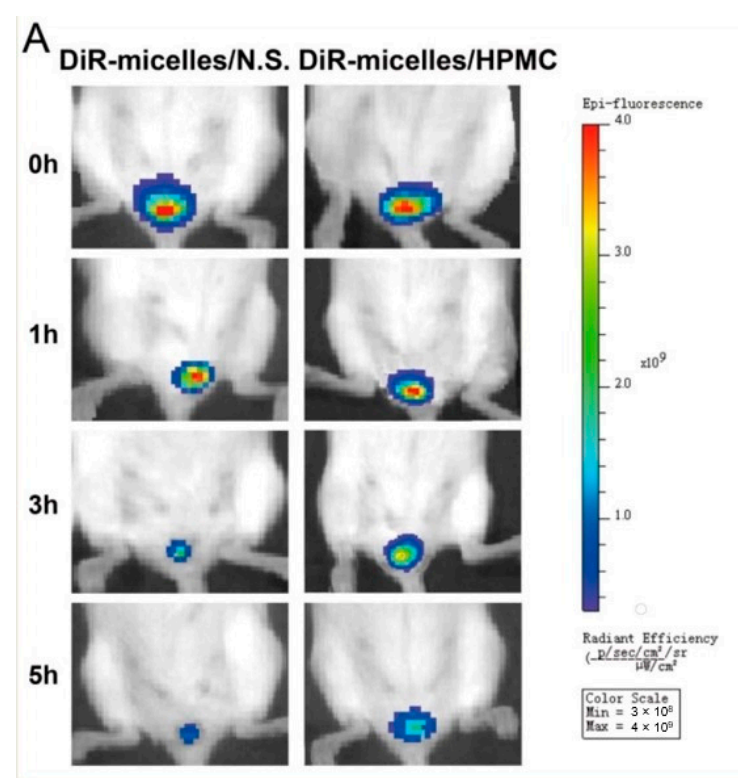
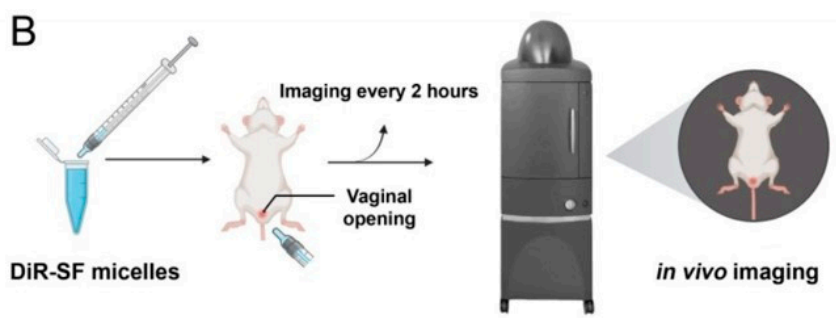

C

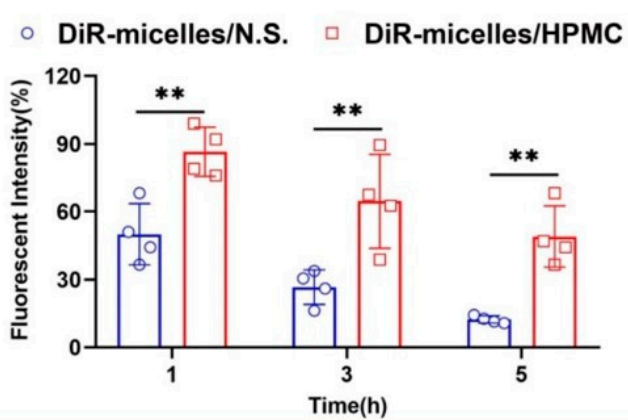

Figure 6. In vivo vaginal retention of SF micelles/N.S. or SF micelles/HPMC after vaginal administration of DiR-labelled SF micelles/N.S. (DiR-micelles/N.S.) or DiR-labelled SF micelles/HPMC (DiR-micelles/ HPMC): (A) Fluorescent images. (B) Schematic diagram of the experimental process. (C) Semi-quantitative fluorescent intensity ( $\mathrm{n}=4$, mean \pm SD). Significance criteria: ${ }^{* *} p<0.01$. 


\subsection{Tumor Shrinkage Effect in the Orthotopic TC-1 Cervical Cancer Model}

It was reported that the orthotopic TC-1 model strictly represents the cervicovaginal cancer in a short time [48]. The bioluminescence images of tumor-bearing mice were shown in Figures 7 and A5, and there was a near linear correlation between bioluminescence signal and tumor weight as reported [49-51], indicating that the bioluminescence TC-1 tumor model could accurately assess tumor size. On the 16th day after intravaginal administration, the bioluminescence intensity of the N.S., HPMC and Empty micelles/HPMC was approximately 155, 156 and 160 times higher than that at day 0 (Figure 7A,B), respectively, with no significant difference between the three groups. The bioluminescence intensity of the Free SF/HPMC group was about 120 times higher than that at day 0, while SF micelles/HPMC was about 76 times higher than that at day 0 . The average tumor weight of $\mathrm{SF}$ micelles/HPMC was $1.27 \mathrm{~g}$ (Figure 7C), the inhibition rate of tumor weight was $72.77 \%$ (Figure A7), and the tumor photographs showed that SF micelles/HPMC had a better tumor shrinkage effect than the Free SF/HPMC (Figure A6). These results indicated that SF micelles/HPMC inhibited tumor growth to the highest extent. There was no significant difference in body weight for all groups (Figure 7D). No obvious difference was observed in H\&E stained-section of major organs among all groups (Figure A8), H\&E stained sections of the vagina in the Free SF group showed that the mucosal epithelium was destroyed, while the mucosal structure in the SF micelles group was intact (Figure 7E), indicating that SF micelles had no significant toxic side effects. All data indicated that SF micelles could safely and effectively inhibit tumor growth.

We noticed that before day 8 , there was no significant difference in the bioluminescence intensity between Free SF and SF micelles, whereas after day 8, Free SF was less effective in inhibiting tumor growth compared with SF micelles because as TC-1 model tumor grew, elevated interstitial pressure within tumor impaired the penetration of free drugs, making it difficult for the Free SF to further reduce the tumor volume [52].

\subsection{Discussion}

In this study, SF micelles were formulated by the film hydration method [37,53], inspired by the " $\mathrm{CO}_{2}$-assisted method", a method that using impetus of $\mathrm{CO}_{2}$ bubbles to reduce the particle size [54], we immersed the nitrogen blowing needle into the liquid during the hydration process, and used the $\mathrm{N}_{2}$ bubbles to achieve rapid micro-mixing, resulting in a uniform distribution of SF and TPGS in the hydration solution, thus facilitating the acquisition of SF micelles with homogenous particle size. After hydration, a probe ultrasonic process was adopted to increase the chance of interaction between the hydrophobic end of TPGS and the hydrophobic drug SF by using ultrasound input energy for strong mixing, and then to increase the drug loading.

The micelles with particle size less than $30 \mathrm{~nm}$ were formed by self-assembly of amphiphilic properties, while the particle size in this study was larger than $50 \mathrm{~nm}$. Considering that SF micelles were prepared using probe ultrasound, it has been reported that ultrasound can break the micelles into smaller micelles fragments and use these fragments as nuclei to promote micelle growth and finally obtain homogeneous micelles [55]. Therefore, SF micelles may be formed by a "micelle aggregation" [56], which aggregated from small simple micelles to form large complex micelles.

The tumor shrinkage effect of Free SF/HPMC was far less than that of SF micelles/ HPMC, which might be due to the hydrophobicity of the Free SF, which easily precipitated in the hydrophilic HPMC environment, and as rapid proliferation of tumor cells, which elevated the interstitial hydraulic pressure and prevented the precipitated particles from entering the dense tumor [51]. In contrast, the hydrophilic chains of TPGS on the surface of SF micelles maintained the stability of SF micelles in HPMC and reduced the adhesion of micelles to mucus, allowing SF micelles to penetrate the mucus to reach the tumor site [57]. 
A

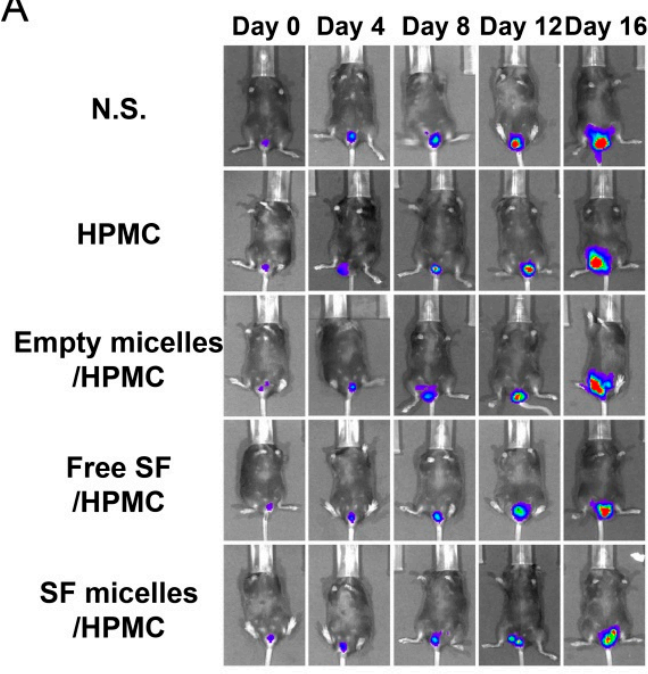

C

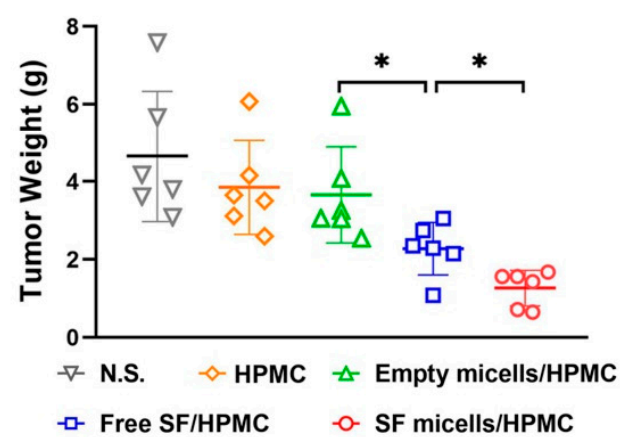

B
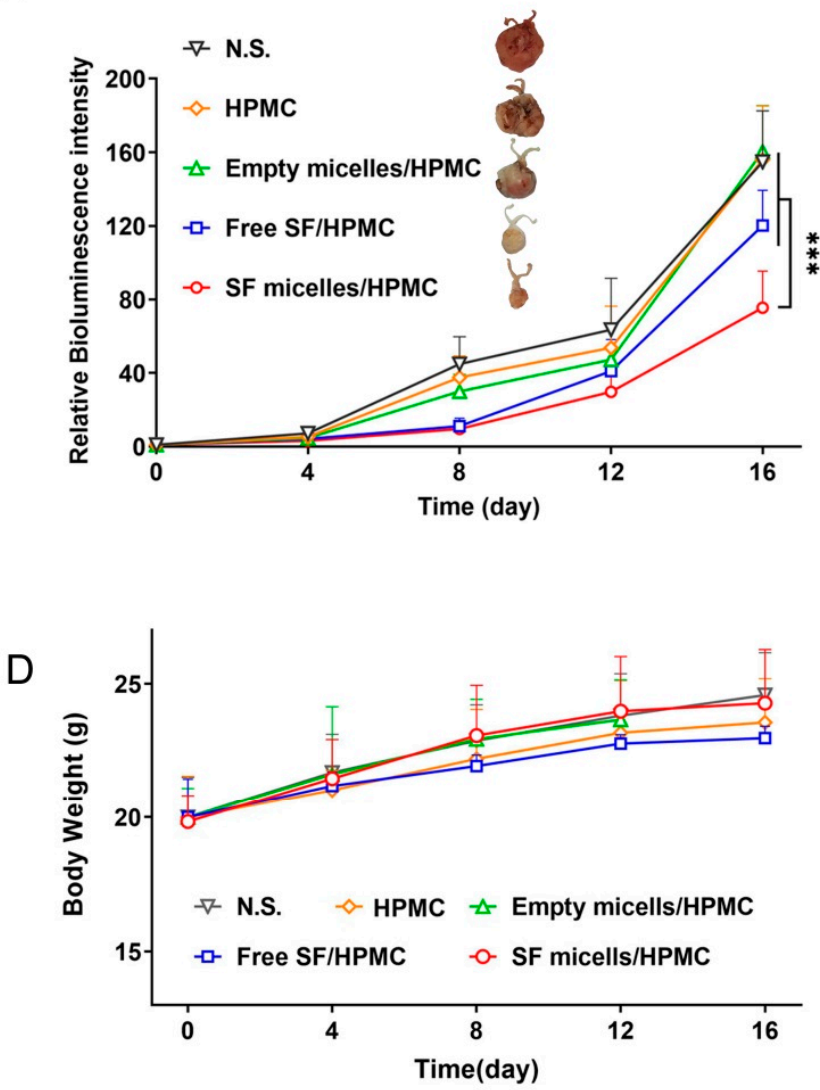

E

N.S.

HPMC
Empty micelles /HPMC
Free SF /HPMC
SF micelles /HPMC

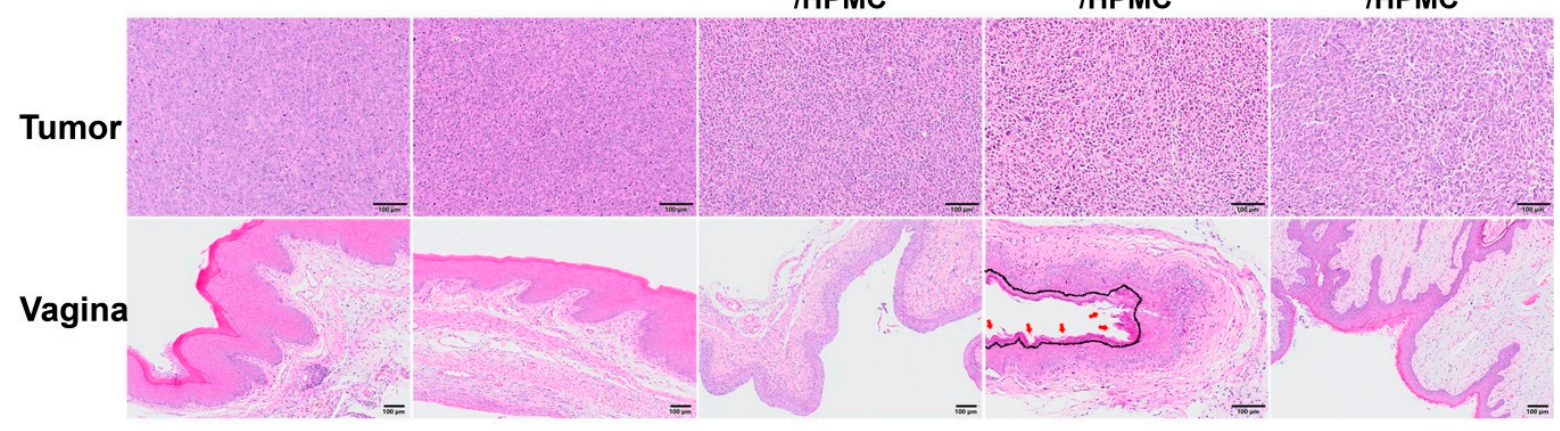

Figure 7. Anti-tumor efficacy of SF micelles/HPMC treatment by intravaginal administration at a dose of $15 \mathrm{mg} / \mathrm{kg}$ in every 3 days for 16 days. (A)Typical bioluminescence images in orthotopic cervical cancer model tumor-bearing mice after intravaginal administration of different formulations at the 0 th, 4 th, 8 th, 12th and 16th day. (B) Relative bioluminescence intensity of tumors (Insert: Typical photographs of orthotopic cervical model tumors by the end of treatment). (C) Tumor weights at the end of treatment $(n=6$, mean $\pm S D)$. (D) Body weights vs. time curves $(n=6$, mean \pm SD). (E) Representative H\&E microphotographs of tumors and vagina. Scale bars $=100 \mu \mathrm{m}$. Significance criteria: ${ }^{*} p<0.05,{ }^{* * *} p<0.001$.

\section{Conclusions}

In conclusion, by using the amphiphilic polymer TPGS as a carrier, we successfully prepared SF micelles that improved the solubility of SF, and showed higher cell uptake and stronger cell growth inhibition compared with Free SF. SF micelles/HPMC exhibited prolonged intravaginal retention in vivo, thus improving the anti-tumor efficacy. Therefore, as a preoperative local chemotherapeutic formulation for cervical cancer, SF micelles/HPMC had a good potential for preoperative tumor shrinkage, which was expected to enhance 
the surgical resection rate of the lesion and improve the postoperative quality of life of the patients.

Author Contributions: Conceptualization, Y.L. and Z.L.; methodology, Y.L., J.W. and F.L.; software, T.S., S.Z. and H.C.; validation, Y.L. and Z.L.; formal analysis, Y.L., J.W. and F.L.; investigation, J.W., F.L., T.S., S.Z. and H.C.; resources, Y.L. and Z.L.; data curation, J.W.; writing-original draft preparation, J.W.; writing—review and editing, J.W.; visualization, Y.L. and Z.L.; supervision, Y.L. and Z.L.; project administration, Y.L. and Z.L.; funding acquisition, Z.L. All authors have read and agreed to the published version of the manuscript.

Funding: This research was funded by the National Key Research and Development Program of China (grant no. 2016YFC1302603) and the National Natural Science Foundation of China (81573361).

Institutional Review Board Statement: The study was approved by the Experimental Animal Ethics Committee, School of Pharmacy, Fudan University (2021-03-SL-LZP-31).

Informed Consent Statement: Not applicable.

Data Availability Statement: Not applicable.

Conflicts of Interest: The authors declare no conflict of interest.

\section{Appendix A}

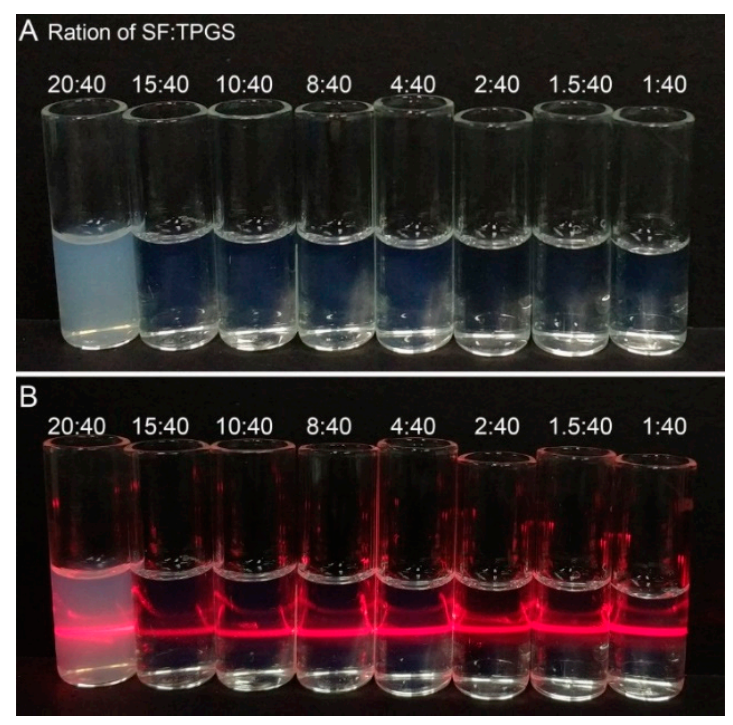

Figure A1. Photographs of (A) SF micelles with different mass ratios of SF:TPGS in glass bottles; (B) Tyndall effects of SF micelles solution.
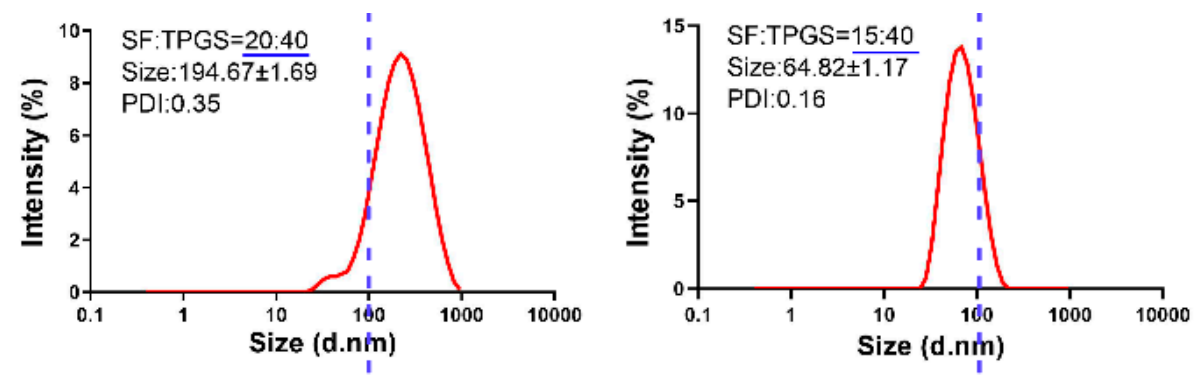

Figure A2. Cont. 

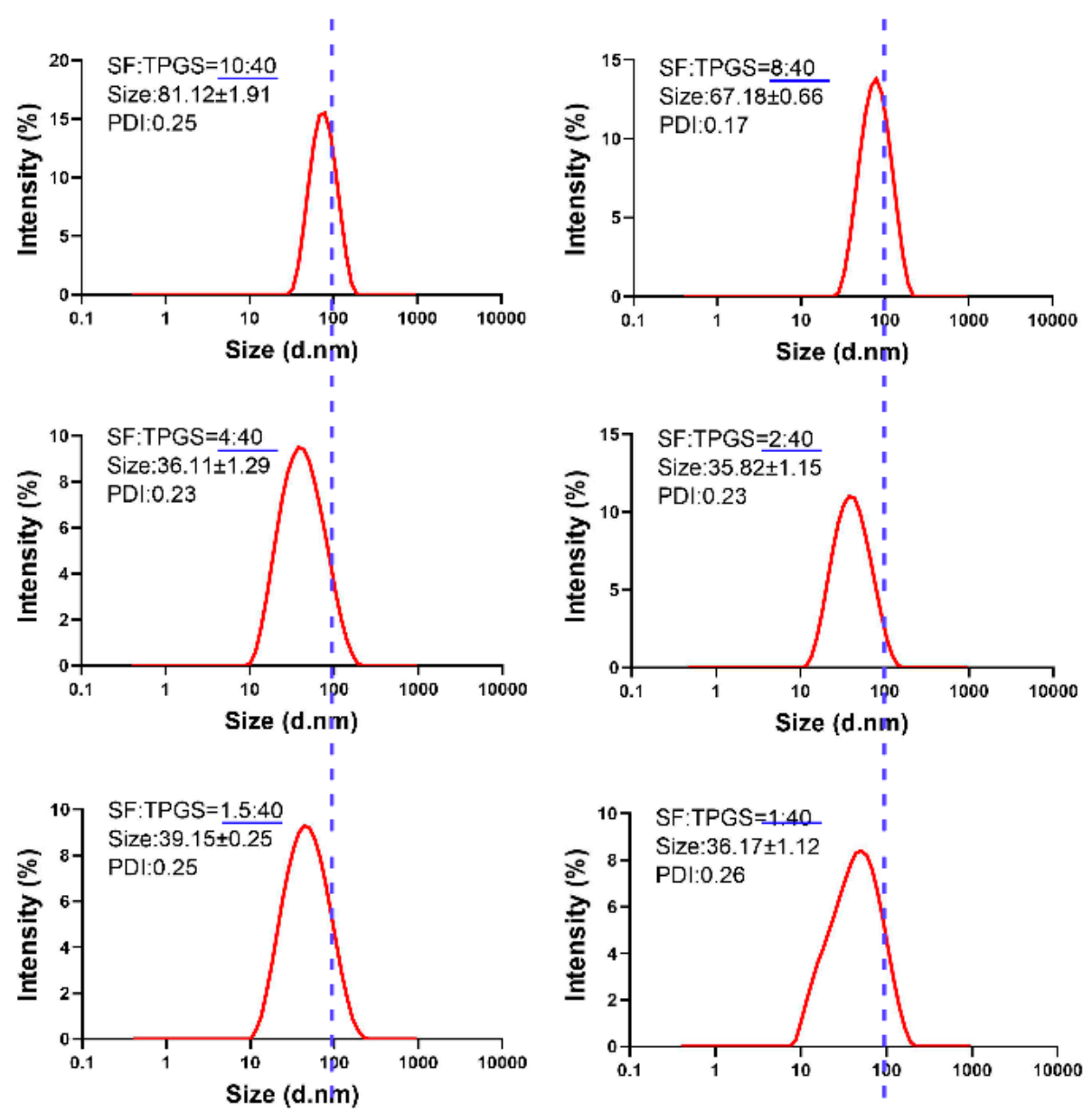

Figure A2. Size distribution of SF micelles with different mass ratios of SF:TPGS measured by DLS.

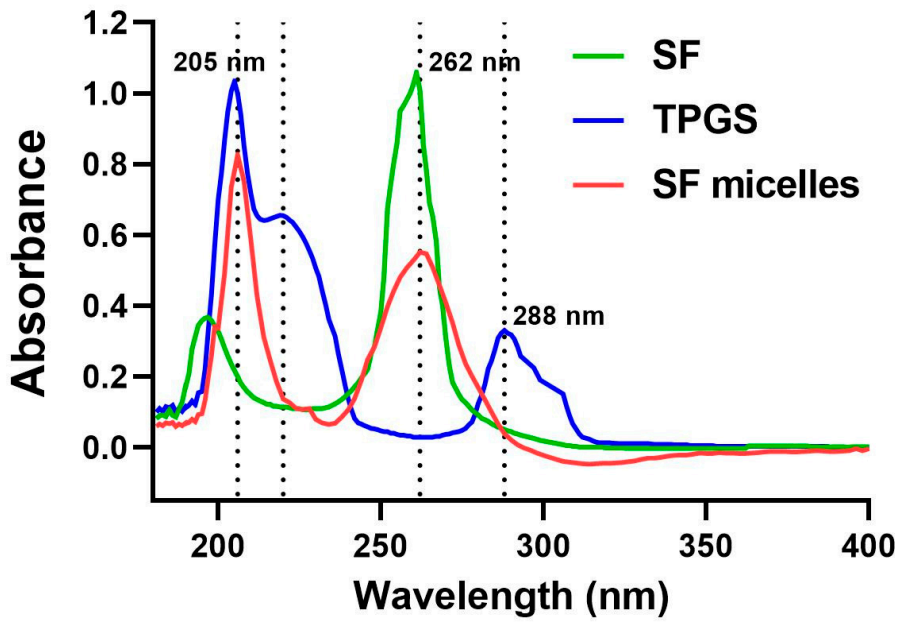

Figure A3. Ultraviolet scanning spectrum of SF, TPGS and SF micelles. 
Table A1. Characterization of SF micelles with different mass ratios of SF:TPGS ( $n=3$, mean \pm SD).

\begin{tabular}{ccccccc}
\hline Sample & SF:TPGS & Size $(\mathbf{n m})$ & PDI & Zeta potential $(\mathbf{m V})$ & DL $(\%)$ & EE $(\%)$ \\
\hline S1 & $20: 40$ & $194.67 \pm 1.69$ & $0.35 \pm 0.002$ & $-2.01 \pm 0.81$ & $30.52 \pm 0.48$ & $57.97 \pm 0.12$ \\
S2 & $15: 40$ & $64.82 \pm 1.17$ & $0.16 \pm 0.003$ & $-1.43 \pm 0.43$ & $23.15 \pm 0.62$ & $63.92 \pm 0.15$ \\
S3 & $10: 40$ & $81.12 \pm 1.91$ & $0.25 \pm 0.014$ & $-1.03 \pm 0.52$ & $18.38 \pm 0.56$ & $70.11 \pm 0.21$ \\
S4 & $8: 40$ & $67.18 \pm 0.66$ & $0.17 \pm 0.008$ & $-1.54 \pm 0.8$ & $15.90 \pm 0.46$ & $89.4 \pm 0.19$ \\
S5 & $4: 40$ & $36.11 \pm 1.29$ & $0.23 \pm 0.006$ & $-2.24 \pm 0.89$ & $8.21 \pm 0.33$ & $90.4 \pm 0.28$ \\
S6 & $2: 40$ & $35.82 \pm 1.15$ & $0.23 \pm 0.004$ & $-0.94 \pm 1.33$ & $3.92 \pm 0.14$ & $90.68 \pm 0.23$ \\
S7 & $1.5: 40$ & $39.15 \pm 0.25$ & $0.25 \pm 0.004$ & $-2.01 \pm 0.44$ & $2.47 \pm 0.19$ & $91.56 \pm 0.13$ \\
S8 & $1: 40$ & $36.17 \pm 1.12$ & $0.26 \pm 0.009$ & $-2.78 \pm 0.34$ & $1.91 \pm 0.21$ & $93.47 \pm 0.32$ \\
\hline
\end{tabular}

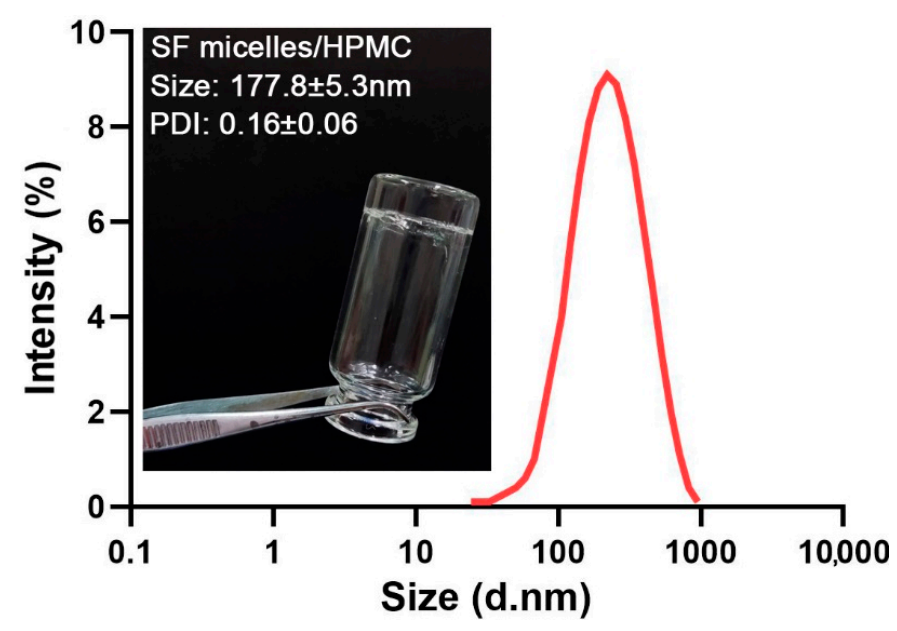

Figure A4. Size distribution of SF micelles dispersed in HPMC.
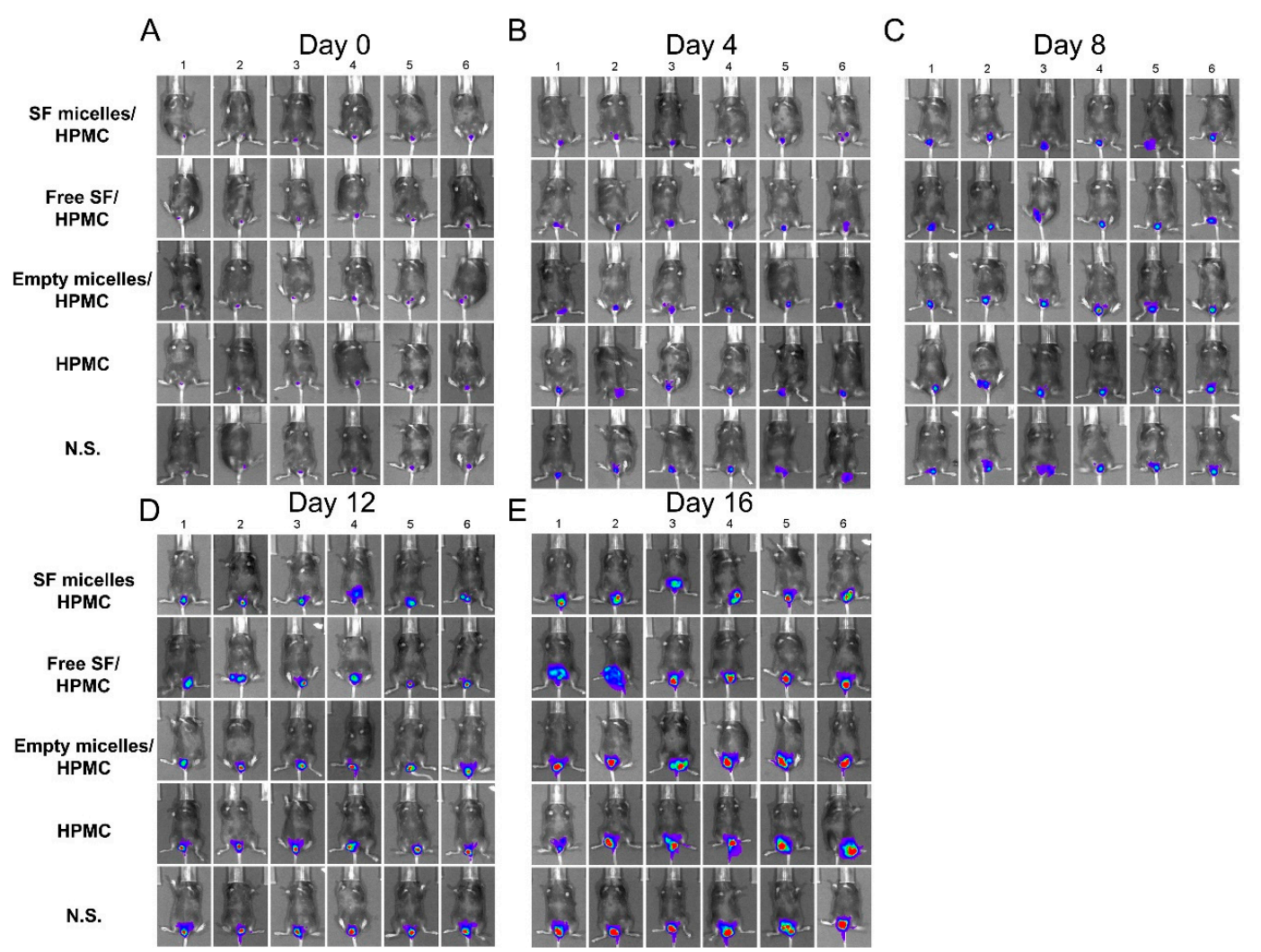

Figure A5. The bioluminescence images of SF micelles/HPMC, Free SF/HPMC, Empty micelles/HPMC, HPMC and N.S. group to the tumor-bearing mice at the day 0 , day 4 , day 8 , day 12 and day16 $(n=6)(\mathbf{A}-\mathbf{E})$. 
N.S.
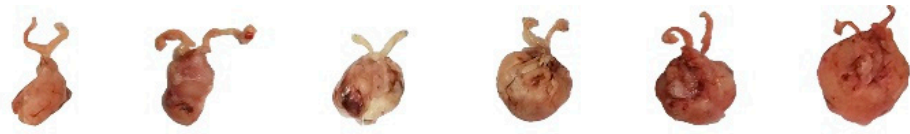

HPMC

\section{然。}
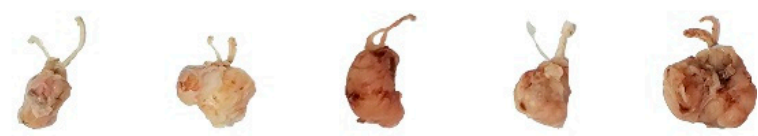

Empty micelles/HPMC
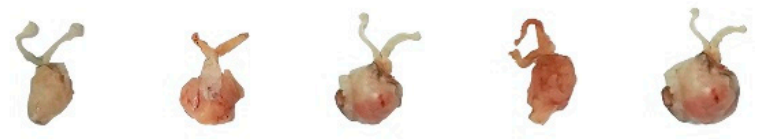

Free SF/HPMC
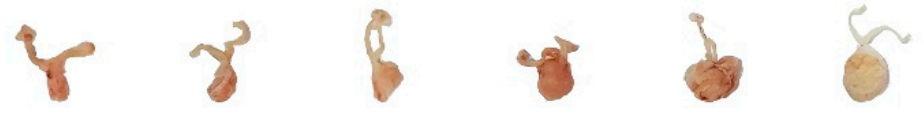

SF micelles/HPMC
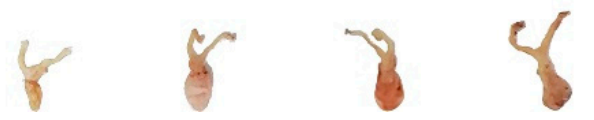

Figure A6. Photographs of orthotopic cervical carcinoma connecting uterus and uterine tubes at the end of treatment.

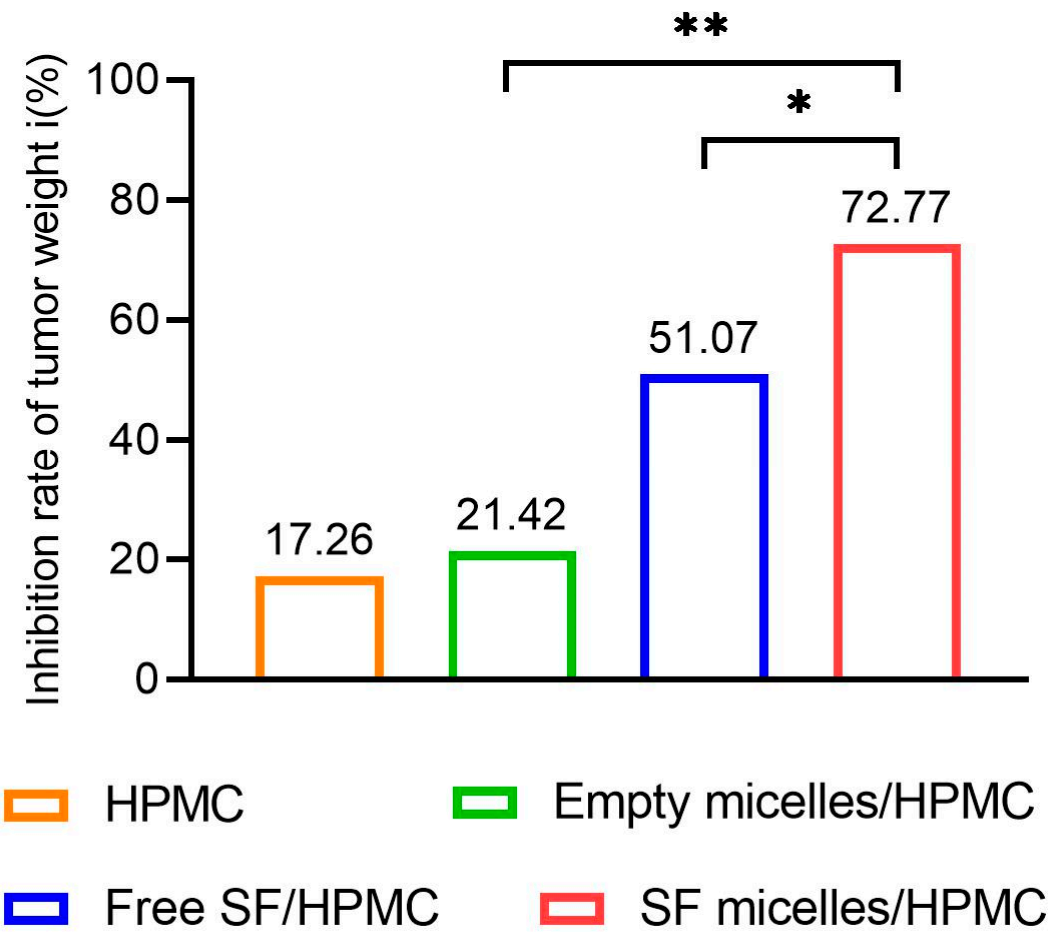

Figure A7. Inhibition rate of tumor weight by the end of treatment. Significance criteria: ${ }^{*} p<0.05$, ** $p<0.001$ 


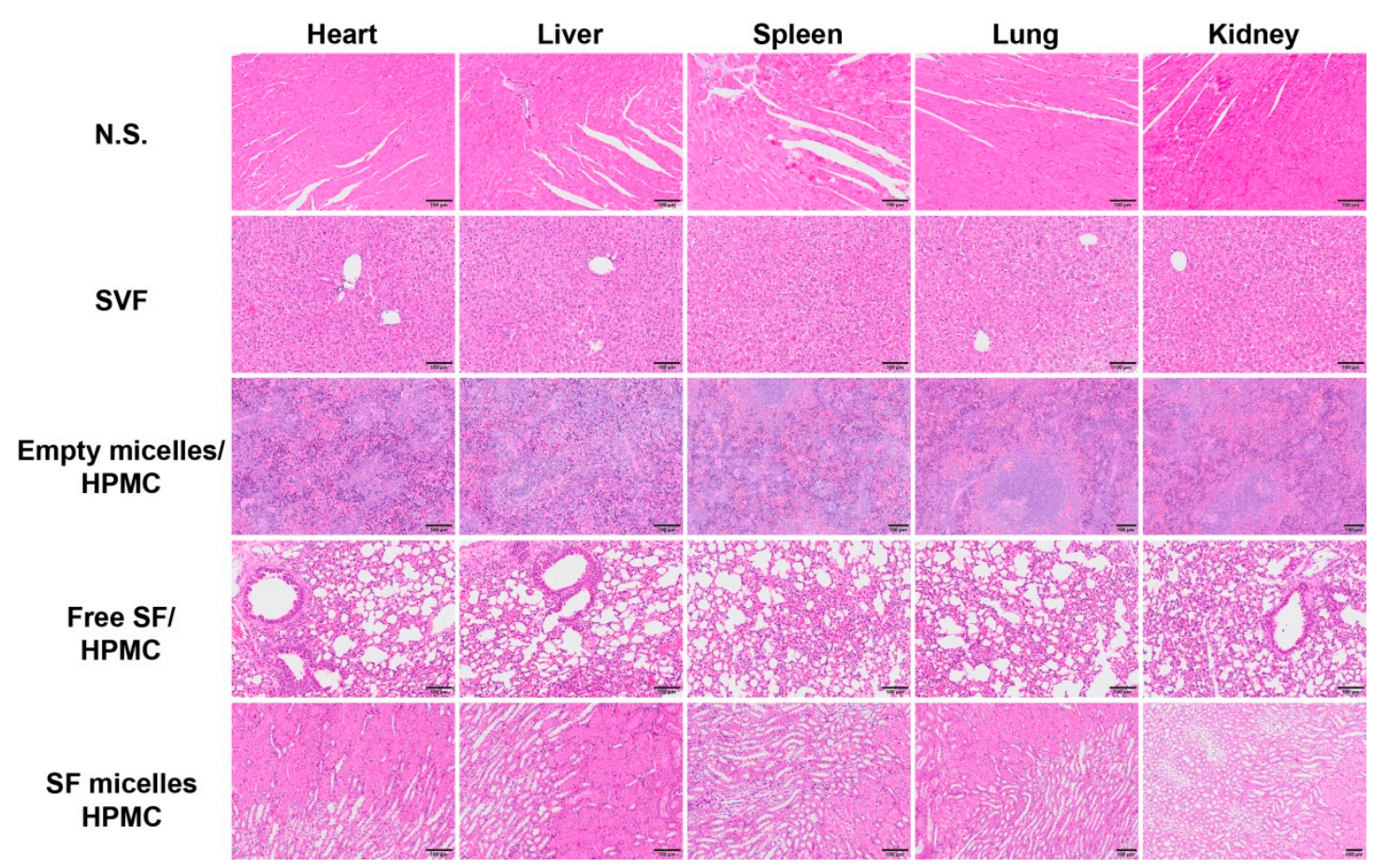

Figure A8. H\&E-stained microscopic images of major organs of mice by the end of treatment (intravaginal administration every 3 days for continuous 16 days at $S F$ dose of $15 \mathrm{mg} / \mathrm{kg} / 3 \mathrm{~d}$ ). Scale bars $=100 \mu \mathrm{m}$.

\section{References}

1. Milosevic, M.F.; Townsley, C.A.; Chaudary, N.; Clarke, B.; Pintilie, M.; Fan, S.; Glicksman, R.; Haider, M.; Kim, S.; MacKay, H.; et al. Sorafenib Increases Tumor Hypoxia in Cervical Cancer Patients Treated With Radiation Therapy: Results of a Phase 1 Clinical Study. Int. J. Radiat. Oncol Biol. Phys. 2016, 94, 111-117. [CrossRef]

2. Katsumata, N.; Yoshikawa, H.; Kobayashi, H.; Saito, T.; Kuzuya, K.; Nakanishi, T.; Yasugi, T.; Yaegashi, N.; Yokota, H.; Kodama, S.; et al. Phase III randomised controlled trial of neoadjuvant chemotherapy plus radical surgery vs radical surgery alone for stages IB2, IIA2, and IIB cervical cancer: A Japan Clinical Oncology Group trial (JCOG 0102). Br. J. Cancer 2013, 108, $1957-1963$. [CrossRef] [PubMed]

3. Yee, G.P.; de Souza, P.; Khachigian, L.M. Current and potential treatments for cervical cancer. Curr. Cancer Drug Targets 2013, 13, 205-220. [CrossRef] [PubMed]

4. Pectasides, D.; Kamposioras, K.; Papaxoinis, G.; Pectasides, E. Chemotherapy for recurrent cervical cancer. Cancer Treat. Rev. 2008, 34, 603-613. [CrossRef] [PubMed]

5. Sardi, J.E.; Boixadera, M.A.; Sardi, J.J. Neoadjuvant chemotherapy in cervical cancer: A new trend. Curr. Opin. Obs. Gynecol. 2005, 17, 43-47. [CrossRef] [PubMed]

6. Tao, X.; Hu, W.; Ramirez, P.T.; Kavanagh, J.J. Chemotherapy for recurrent and metastatic cervical cancer. Gynecol. Oncol. 2008, 110, S67-S71. [CrossRef]

7. Markman, M. Advances in cervical cancer pharmacotherapies. Expert Rev. Clin. Pharmacol. 2014, 7, 219-223. [CrossRef]

8. Sun, W.; Wang, X.; Li, J.; You, C.; Lu, P.; Feng, H.; Kong, Y.; Zhang, H.; Liu, Y.; Jiao, R.; et al. MicroRNA-181a promotes angiogenesis in colorectal cancer by targeting SRCIN1 to promote the SRC/VEGF signaling pathway. Cell Death Dis. $2018,9,438$. [CrossRef]

9. Ma, M.K.F.; Lau, E.Y.T.; Leung, D.H.W.; Lo, J.; Ho, N.P.Y.; Cheng, L.K.W.; Ma, S.; Lin, C.H.; Copland, J.A.; Ding, J.; et al. Stearoyl-CoA desaturase regulates sorafenib resistance via modulation of ER stress-induced differentiation. J. Hepatol. 2017, 67, 979-990. [CrossRef] [PubMed]

10. del Campo, J.M.; Prat, A.; Gil-Moreno, A.; Perez, J.; Parera, M. Update on novel therapeutic agents for cervical cancer. Gynecol Oncol. 2008, 110, S72-S76. [CrossRef] [PubMed]

11. Bray, F.; Ferlay, J.; Soerjomataram, I.; Siegel, R.L.; Jemal, A. Global Cancer Statistics 2018: GLOBOCAN Estimates of Incidence and Mortality Worldwide for 36 Cancers in 185 Countries. CA Cancer J. Clin. 2018, 68, 394-424. [CrossRef]

12. Gadaleta, C.D.; Sciorsci, R.L.; Rizzo, A.; Mangia, A.; Patruno, R.; Goffredo, V.; Gadaleta-Caldarola, G.; Ranieri, G. Sorafenib (BAY 43-9006) in hepatocellular carcinoma patients: From discovery to clinical development. Curr. Med. Chem. 2012, 19, 938-944.

13. Liu, C.; Chen, Z.; Chen, Y.; Lu, J.; Li, Y.; Wang, S.; Wu, G.; Qian, F. Improving Oral Bioavailability of Sorafenib by Optimizing the "Spring" and "Parachute" Based on Molecular Interaction Mechanisms. Mol. Pharm. 2016, 13, 599-608. [CrossRef] [PubMed] 
14. Ganten, T.M.; Stauber, R.E.; Schott, E.; Malfertheiner, P.; Buder, R.; Galle, P.R.; Göhler, T.; Walther, M.; Koschny, R.; Gerken, G. Sorafenib in Patients with Hepatocellular Carcinoma-Results of the Observational INSIGHT Study. Clin. Cancer Res. 2017, 23, 5720-5728. [CrossRef] [PubMed]

15. Pellosi, D.S.; Moret, F.; Fraix, A.; Marino, N.; Maiolino, S.; Gaio, E.; Hioka, N.; Reddi, E.; Sortino, S.; Quaglia, F. Pluronic ${ }^{\circledR}$ P123/F127 mixed micelles delivering sorafenib and its combination with verteporfin in cancer cells. Int. J. Nanomed. 1988, 11, 4479 .

16. Zhang, L.; Gong, F.; Zhang, F.; Ma, J.; Zhang, P.; Shen, J. Targeted therapy for human hepatic carcinoma cells using folatefunctionalized polymeric micelles loaded with superparamagnetic iron oxide and sorafenib in vitro. Int. J. Nanomed. 2013, 2013, 1517-1524. [CrossRef] [PubMed]

17. Su, Y.; Wang, K.; Li, Y.; Song, W.; Xin, Y.; Zhao, W.; Tian, J.; Ren, L.; Lu, L. Sorafenib-loaded polymeric micelles as passive targeting therapeutic agents for hepatocellular carcinoma therapy. Nanomedicine 2018, 13, 1009-1023. [CrossRef] [PubMed]

18. Mo, L.; Song, J.G.; Lee, H.; Zhao, M.; Kim, H.Y.; Lee, Y.J.; Ko, H.W.; Han, H.K. PEGylated hyaluronic acid-coated liposome for enhanced in vivo efficacy of sorafenib via active tumor cell targeting and prolonged systemic exposure. Nanomed. Nanotechnol. Biol. Med. 2018, 14, 557-567. [CrossRef]

19. Zhao, M.; Lee, S.H.; Song, J.G.; Kim, H.Y.; Han, H.K. Enhanced oral absorption of sorafenib via the layer-by-layer deposition of a $\mathrm{pH}$-sensitive polymer and glycol chitosan on the liposome. Int. J. Pharm. 2018, 544, 14-20. [CrossRef]

20. Liu, J.; Boonkaew, B.; Arora, J.; Mandava, S.H.; Maddox, M.M.; Chava, S.; Callaghan, C.; He, J.; Dash, S.; John, V.T.; et al. Comparison of Sorafenib-Loaded Poly (Lactic/Glycolic) Acid and DPPC Liposome Nanoparticles in the in Vitro Treatment of Renal Cell Carcinoma. J. Pharm. Sci. 2015, 104, 1187-1196. [CrossRef]

21. Giglio, V.; Viale, M.; Bertone, V.; Maric, I.; Vaccarone, R.; Vecchio, G. Cyclodextrin polymers as nanocarriers for sorafenib. Investig. New Drugs 2018, 36, 370-379. [CrossRef] [PubMed]

22. Guo, Y.; Zhong, T.; Duan, X.C.; Zhang, S.; Yao, X.; Yin, Y.F.; Huang, D.; Ren, W.; Zhang, Q.; Zhang, X. Improving anti-tumor activity of sorafenib tosylate by lipid- and polymer-coated nanomatrix. Drug Deliv. 2017, 24, 270-277. [CrossRef] [PubMed]

23. Bernabeu, E.; Chiappetta, D.A. Vitamin E TPGS Used as Emulsifier in the Preparation of Nanoparticulate Systems. J. Biomater. Tissue Eng. 2013, 3, 122-134. [CrossRef]

24. Dou, J.; Zhang, H.; Liu, X.; Zhang, M.; Zhai, G. Preparation and evaluation in vitro and in vivo of docetaxel loaded mixed micelles for oral administration. Colloids Surf. B Biointerfaces 2014, 114, 20-27. [CrossRef] [PubMed]

25. Zhang, X.Y.; Zhang, Y.D. Enhanced antiproliferative and apoptosis effect of paclitaxel-loaded polymeric micelles against non-small cell lung cancers. Tumour Biol. J. Int. Soc. Oncodev. Biol. Med. 2015, 36, 4949. [CrossRef]

26. Muthu, M.S.; Kulkarni, S.A.; Liu, Y.; Feng, S.S. Development of docetaxel-loaded vitamin E TPGS micelles: Formulation optimization, effects on brain cancer cells and biodistribution in rats. Nanomedicine 2012, 7, 353-364. [CrossRef] [PubMed]

27. Zhang, Z.; Tan, S.; Feng, S.S. Vitamin E TPGS as a molecular biomaterial for drug delivery. Biomaterials 2012, 33, 4889-4906. [CrossRef]

28. Tuğcu-Demiröz, F.; Acartürk, F.; Erdoğan, D. Development of long-acting bioadhesive vaginal gels of oxybutynin: Formulation, in vitro and in vivo evaluations. Int. J. Pharm. 2013, 457, 25-39. [CrossRef] [PubMed]

29. Owen, D.H.; Katz, D.F. A vaginal fluid simulant. Contraception 1999, 59, 91-95. [CrossRef]

30. Yang, M.; Yu, T.; Wang, Y.Y.; Lai, S.K.; Zeng, Q.; Miao, B.; Tang, B.C.; Simons, B.W.; Ensign, L.M.; Liu, G.; et al. Vaginal Delivery of Paclitaxel via Nanoparticles with Non-Mucoadhesive Surfaces Suppresses Cervical Tumor Growth. Adv. Healthc. Mater. 2014, 3, 1044-1052. [CrossRef]

31. Zhang, J.; Wang, T.; Mu, S.; Olerile, L.D.; Yu, X.; Zhang, N. Biomacromolecule/lipid hybrid nanoparticles for controlled delivery of sorafenib in targeting hepatocellular carcinoma therapy. Nanomedicine 2017, 12, 911-925. [CrossRef] [PubMed]

32. Ci, L.; Huang, Z.; Yu, L.; Liu, Z.; Gang, W.; Lu, W. Amino-functionalized poloxamer 407 with both mucoadhesive and thermosensitive properties:preparation, characterization and application in a vaginal drug delivery system. Acta Pharm. Sin. B. 2017, 7, 593-602. [CrossRef] [PubMed]

33. Ci, L.Q.; Huang, Z.G.; Lv, F.M.; Wang, J.; Feng, L.L.; Sun, F.; Cao, S.J.; Liu, Z.P.; Liu, Y.; Wei, G.; et al. Enhanced Delivery of Imatinib into Vaginal Mucosa via a New Positively Charged Nanocrystal-Loaded in Situ Hydrogel Formulation for Treatment of Cervical Cancer. Pharmaceutics 2019, 11, 15. [CrossRef]

34. Jug, M.; Hafner, A.; Lovrić, J.; Kregar, M.L.; Pepić, I.; Vanić, Ž.; Cetina-Čižmek, B.; Filipović-Grčić, J. An overview of in vitro dissolution/release methods for novel mucosal drug delivery systems. J. Pharm. Biomed. Anal. 2018, 147, 350-366. [CrossRef] [PubMed]

35. Paveli, E.; Skalko-Basnet, N.; Filipovi-Gri, J.; Martinac, A.; Jalšenjak, I. Development and in vitro evaluation of a liposomal vaginal delivery system for acyclovir. J. Control. Release 2005, 106, 34-43. [CrossRef] [PubMed]

36. Zierden, H.C.; Josyula, A.; Shapiro, R.L.; Hsueh, H.T.; Ensign, L.M. Avoiding a Sticky Situation: Bypassing the Mucus Barrier for Improved Local Drug Delivery. Trends Mol. Med. 2021, 27, 436-450. [CrossRef]

37. Kim, M.S.; Kim, J.S.; Cho, W.K.; Hwang, S.J. Enhanced solubility and oral absorption of sirolimus using D- $\alpha$-tocopheryl polyethylene glycol succinate micelles. Artif. Cells 2013, 41, 85-91. [CrossRef]

38. Lu, Y.; Li, Y.; Wu, W. Injected nanocrystals for targeted drug delivery. Acta Pharm. Sin. B. 2016, 6, 106-113. [CrossRef] [PubMed] 
39. Popielarski, S.R.; Hu-Lieskovan, S.; French, S.W.; Triche, T.J.; Davis, M.E. A Nanoparticle-Based Model Delivery System To Guide the Rational Design of Gene Delivery to the Liver. 2. In Vitro and In Vivo Uptake Results. Bioconjug. Chem. 2005, 16, 1071-1080. [CrossRef]

40. Madathiparambil Visalakshan, R.; González García, L.E.; Benzigar, M.R.; Ghazaryan, A.; Simon, J.; Mierczynska-Vasilev, A.; Michl, T.D.; Vinu, A.; Mailänder, V.; Morsbach, S.; et al. The Influence of Nanoparticle Shape on Protein Corona Formation. Small 2020, 16, e2000285. [CrossRef]

41. Chai, Z.; Ran, D.; Lu, L.; Zhan, C.; Ruan, H.; Lu, X.; Xie, C.; Jiang, K.; Li, J.; Zhou, J.; et al. Ligand-Modified Cell Membrane Enables Targeted Delivery of Drug Nanocrystals to Glioma. ACS Nano 2019, 13, 5591-5601. [CrossRef]

42. Wang, J.; Lv, F.M.; Wang, D.L.; Du, J.L.; Guo, H.Y.; Chen, H.N.; Zhao, S.J.; Liu, Z.P.; Liu, Y. Synergistic Antitumor Effects on Drug-Resistant Breast Cancer of Paclitaxel/Lapatinib Composite Nanocrystals. Molecules 2020, 25, 604. [CrossRef] [PubMed]

43. Mi, Y.; Mu, C.; Wolfram, J.; Deng, Z.; Hu, T.Y.; Liu, X.; Blanco, E.; Shen, H.; Ferrari, M. A Micro/Nano Composite for Combination Treatment of Melanoma Lung Metastasis. Adv Healthc Mater. 2016, 5, 936-946. [CrossRef]

44. Yu, C.; He, B.; Zhang, H.; Dai, W.B.; Wang, X.Q.; Zhang, Q. Transcellular process of coumarin 6 loaded PEG-PCL micelles on MDCK epithelial cells. Acta Pharm Sin. 2013, 48, 1484-1490.

45. Valenta, C. The use of mucoadhesive polymers in vaginal delivery. Adv Drug Deliv Rev. 2005, 57, 1692-1712. [CrossRef] [PubMed]

46. Lai, S.K.; O'Hanlon, D.E.; Harrold, S.; Man, S.T.; Wang, Y.Y.; Cone, R.; Hanes, J. Rapid transport of large polymeric nanoparticles in fresh undiluted human mucus. Proc. Natl. Acad. Sci. USA 2007, 104, 1482-1487. [CrossRef] [PubMed]

47. Lv, F.; Wang, J.; Chen, H.; Sui, L.; Feng, L.; Liu, Z.; Liu, Y.; Wei, G.; Lu, W. Enhanced mucosal penetration and efficient inhibition efficacy against cervical cancer of PEGylated docetaxel nanocrystals by TAT modification. J Control. Release 2021, 336, 572-582. [CrossRef]

48. Zeng, Q.; Peng, S.; Monie, A.; Yang, M.; Pang, X.; Hung, C.F.; Wu, T.C. Control of Cervicovaginal HPV-16 E7-Expressing Tumors by the Combination of Therapeutic HPV Vaccination and Vascular Disrupting Agents. Hum Gene Ther. 2011, 22, 809-819. [CrossRef] [PubMed]

49. Scatena, C.D.; Hepner, M.A.; Oei, Y.A.; Dusich, J.M.; Yu, S.F.; Purchio, T.; Contag, P.R.; Jenkins, D.E. Imaging of bioluminescent LNCaP-luc-M6 tumors: A new animal model for the study of metastatic human prostate cancer. Prostate 2010, 59, 292-303. [CrossRef]

50. Edinger, M.; Cao, Y.A.; Hornig, Y.S.; Jenkins, D.E.; Verneris, M.R.; Bachmann, M.H.; Negrin, R.; Contag, C. Advancing animal models of neoplasia through in vivo bioluminescence imaging. Eur. J. Cancer 2002, 38, 2128-2136. [CrossRef]

51. Zeamari, S.; Rumping, G.; Floot, B.; Lyons, S.; Stewart, F.A. In vivo bioluminescence imaging of locally disseminated colon carcinoma in rats. Br. J. Cancer. 2004, 90, 1259-1264. [CrossRef]

52. Lin, K.Y.; Guarnieri, F.G.; Staveley-O'Carroll, K.F.; Levitsky, H.I.; August, J.T.; Pardoll, D.M.; Wu, T.C. Treatment of established tumors with a novel vaccine that enhances major histocompatibility class II presentation of tumor antigen. Cancer Res. 1996, 56, 21-26. [PubMed]

53. Yan, G.; Ling, B.L.; Zhai, G. Preparation and characterization of Pluronic/TPGS mixed micelles for solubilization of camptothecin. Colloids Surf. B Biointerfaces 2008, 64, 194-199.

54. Wang, Y.; Han, X.; Wang, J.; Wang, Y. Preparation, characterization and in vivo evaluation of amorphous tacrolimus nanosuspensions produced using CO2-assisted in situ nanoamorphization method. Int. J. Pharm. 2016, 505, 35-41. [CrossRef] [PubMed]

55. Song, S.; Liu, X.; Nikbin, E.; Howe, J.Y.; Yu, Q.; Manners, I.; Winnik, M.A. Uniform 1D Micelles and Patchy And Block Comicelles via Scalable, One-Step Crystallization-Driven Block Copolymer Self-Assembly. J. Am. Chem. Soc. 2021, 143, 6266-6280. [CrossRef] [PubMed]

56. Qi, M.; Zhou, Y. Multimicelle aggregate mechanism for spherical multimolecular micelles: From theories, characteristics and properties to applications. Mater. Chem. Front. 2019, 3, 1994-2009. [CrossRef]

57. Ensign, L.M.; Schneider, C.; Suk, J.S.; Cone, R.; Hanes, J. Mucus penetrating nanoparticles: Biophysical tool and method of drug and gene delivery. Adv Mater. 2012, 24, 3887-3894. [CrossRef] [PubMed] 